\title{
Explaining the "Buy One Get One Free" Promotion: The Golden Ratio as a Marketing Tool
}

\author{
Philip Thomas ${ }^{1}$, Alec Chrystal ${ }^{2}$ \\ ${ }^{1}$ School of Engineering and Mathematical Sciences, City University London, London, UK; ${ }^{2}$ Cass Business School, City University \\ London, London, UK. \\ Email: pjt3.michaelmas@gmail.com
}

Received October $20^{\text {th }}, 2013$; revised November $20^{\text {th }}, 2013$; accepted November $27^{\text {th }}, 2013$

Copyright (C) 2013 Philip Thomas, Alec Chrystal. This is an open access article distributed under the Creative Commons Attribution License, which permits unrestricted use, distribution, and reproduction in any medium, provided the original work is properly cited. In accordance of the Creative Commons Attribution License all Copyrights (C) 2013 are reserved for SCIRP and the owner of the intellectual property Philip Thomas, Alec Chrystal. All Copyright (C) 2013 are guarded by law and by SCIRP as a guardian.

\begin{abstract}
Buy-one-get-one-free (BOGOF) promotions are a common feature of retail food markets, but why are they so widespread? The theory of Relative Utility Pricing (RUP) developed in this paper provides an explanation not only for supermarket promotional offers but also for more general pricing of packs of different sizes in supermarkets and on the internet. A clear and simple explanation is given for the two most widely used quantity promotions: BOGOF and 3 -for-the-price-of-2. The RUP model may be linked to the theory of iso-elastic utility functions, and this allows the relationships amongst risk-aversion, pack-size ratio and demand elasticity to be explored. "Cautious consumers", as defined in the paper, are found to be the only sensible target for quantity promotions. It is argued that the needs of cautious consumers of retail commodities will be best addressed if the vendor sets the ratio of successive pack sizes as the square of the Golden Ratio, namely 2.62, and the price-ratio at the Golden Ratio, 1.62. Thus the Golden Ratio may be regarded as a marketing guide for vendors considering both their best interests and those of their customers. This proposition is supported by an analysis showing that higher profits are more likely to come from Golden Ratio sizing than from either BOGOF or 3-for-2 when variable costs lie in most of the upper half of the range that is required for any of these multibuy offers to generate profit. The paper's theoretical predictions for both pack sizes and prices are supported by examples from the retail sector: grocery, paperback books and electronics.
\end{abstract}

Keywords: Relative Utility Pricing; Golden Ratio Pricing; Buy One Get One Free; Multibuy Promotions; Utility Theory

\section{Introduction}

Retailers often offer products in packs of different sizes, whether it is a food product, electronics or clothing. Moreover, multibuy promotions such as buy-one-getone-free (BOGOF) are common, but why are they so widespread?

Put formally, the research questions addressed by this paper are as follows. Suppose that a pack larger than the entry-level pack is offered, what price will the vendor need to charge in order to convince the consumer that he or she should buy it? Then if further, larger packs are put on the market, what prices will the vendor need to set in order to sell these? What effect will the relative sizes of the packs have on the reaction of the consumers? Finally, how can the retailer maximise his profit given the atti- tudes of the consumer.

\section{Background}

In his 2008 article in The Daily Telegraph, the Consumer Affairs Editor revealed that "more than $80 \%$ of all promotional activity within supermarkets is a bogof [buyone-get-one-free] or three-for-two" [1]. So concerned is the UK Government about the success of such promotions applied to food that it is considering banning multibuy deals in an effort to tackle obesity [2].

But there has long been puzzlement about vendors' motivation in offering quantity promotions. Back in 1984, the Wall Street Journal carried a front-page article stating that: "The dark secrets of packaging aren't always easy to unwrap. While the issue of how much product a com- 
pany should put in a box, bottle or tube is about as basic as any in business, it also can be fraught with complexities" [3]; see also [4].

Tabarrok [5] in his response to Pressler [6] suggests that BOGOFs "generally increase total welfare because the price on the last unit sold is pushed closer to marginal cost and because of this output expands". Certainly, it is relatively easy to understand how vendors will profit from the selling of larger packs of the same good when the marginal cost associated with the extra amount of good is small compared with the fixed costs the retail outlet has to bear (e.g. [7]).

Nevertheless worries have been expressed for some time in academic journals that BOGOF-type promotions might lead people to endanger their health through overeating, e.g. [8]. While, according to Wallop, the distinction between perishable and non-perishable goods means that BOGOFs in supermarkets "are almost twice as popular in the toiletries aisles as in the meat or vegetable section", the same restraint on food offerings does not carry over to fast-food chains. Here Dobson and Gerstner [8] confirm that "supersizing" food portions and offering the larger size at "only a few cents more" than the regular portion can be profitable for the vendor, but claim that this may be harmful to the health of the consumer and, moreover, create economic inefficiencies.

However, no satisfactory economic explanation has been available until now for the reaction of the consumer to the multibuy promotions. It is thus necessary to provide a quantitative explanation for how the larger packs should be sized and priced if the consumer is to be induced to buy a quantity greater than that contained in the standard size, and the logic of these promotions needs to be understood when restrictions are contemplated by the authorities.

The problem is a general one, applying to retail outlets including supermarkets, restaurants, public houses, fastfood stores and internet shopping. It also features in market surveys where the respondent is asked to set a price on different options.

The first part of the paper (up to and including Section 8) will be devoted to developing a model to explain quantitatively the prices consumers will expect for different pack sizes. Utility theory will be used in Sections 3, 4 and 5 to develop the general Relative Utility Pricing (RUP) model, applicable where product differentiation is low and the goods may be regarded as commodities. The model developed provides, inter alia, a theoretical explanation for quantity discounts.

Section 6 develops the RUP model for the common case where, when deliberating whether or not to buy pack $n$, the customer chooses to compare its benefit with the benefit he would gain by buying pack $n-1$. This leads to the Single Comparison Relative Utility Pricing model (SCRUP). The results of this model are surpriseingly simple, and provide a complete explanation of why the retailer will find it necessary to offer buy-one-getone-free (BOGOF) or 3-for-the-price-of-2 promotions. Put colloquially, people will expect "to get $n$ but pay $n-1$ ". The theoretical results are used in Section 7 to explain some actual prices achieved in supermarkets and on the internet, including in cases where the explanation for the pack size and price structure is non-obvious.

Section 8 compares the price per unit produced by the SCRUP model with that produced by applying an isoelastic utility function. It is shown that the natural targets for multibuy promotions are cautious consumers, who have a risk-aversion between 0.0 and 1.0. A link is developed between pack size and the average risk-aversion amongst cautious consumers, which leads to the notion of Golden Ratio Pricing. Here the price quotient of successive packs ratio is the golden ratio while the pack-size ratio is the square of that figure.

Then, in Section 9, an analysis will be made of how the retailer may design his pack 2 offering so as to maximise his profit while satisfying his customers' requirements on price and volume. It will be shown how the retailer may use the knowledge of his variable costs to maximise his profit in his choice of which multibuy offer to make: BOGOF, or 3-for-2 or the new promotion suggested in this paper, Golden Ratio Pricing.

\section{The Utility of Packs of Different Sizes}

Consider a basic good, $B$, that is offered for sale in a number of differently sized packs identified by index, $j$, with $j=0,1,2, \cdots, k, \cdots, n, \cdots, N$, where $j=0$ signifies the empty pack - no purchase is made - and the index, $j$, increases with pack size. Thus $j=1$ indicates the smallest, entry-level pack, while pack $N$ is the largest pack on sale. The indices, $k$ and $n$, with $k<n$, have been introduced to facilitate later comparisons between a general pack, $n$, and a pack, $k$, that is strictly smaller. The maximum ranges of the three indices, $j, k$ and $n$ are:

$$
\begin{aligned}
& 0 \leq j \leq N \\
& 0 \leq k \leq N-1 \\
& 1 \leq n \leq N
\end{aligned}
$$

The amount of good, $B$, in the world may be very large indeed, but we shall be concerned normally with small amounts, $b$, measured in some unit of extent, for example mass or volume or an entirely different measure, such as gigabytes of memory on a USB memory stick.

Let pack $j$ contain an amount, $b_{j}$, of $B$, where, as noted above, $b_{0}=0$ and $b_{j}>b_{j-1}$ for $1 \leq j \leq N$. We will define the largeness, $L(j)$, of pack $j$ as its size relative to the entry-level pack 1 : 


$$
L(j)=\frac{b_{j}}{b_{1}} \quad \text { for } 0 \leq j \leq N
$$

Thus the largeness of pack $j$ is the number of standard units that pack $j$ contains, where a standard unit comprises the contents of pack 1 , namely $b_{1}$. Hence $L(0)=0$ and $L(1)=1$.

Kahneman and Tversky [9] have argued that "people normally perceive outcomes as gains and losses [relative to the current asset position], rather than as final states of wealth or welfare", a proposition supported by Thaler [10]. This approach of measuring the utility of the change in the stock level rather than the utility of the absolute value of the stock is adopted in this paper, and leads to a consideration of the utility of the contents of the pack. Thus the utility of the empty pack, with contents $b_{0}=0$ will be $u\left(b_{0}\right)$, the utility of the contents of pack 1 , namely $b_{1}$, will be $u\left(b_{1}\right)$, and, in general, the purchase of pack $j$ will result in a utility, $u\left(b_{j}\right)$.

Consider the case where the vendor is offering just two packs: pack 1 , with contents, $b_{1}$, and a bigger pack, let us call it pack $x$, with contents, $16 b_{1}$. We will assume that the price of the entry-level pack, pack 1 , has been accepted by purchasers as $p_{1}$, implying that these consumers will have a maximum acceptable price (MAP) of at least $p_{1}$. (See [11] and [12] for a discussion of the concept of maximum acceptable price and probability distributions.)

Let us select one of these purchasers with a MAP of $p_{1}$ or higher, who buys one standard pack. We may give him the name, "consumer X". Meanwhile there may be another person, say consumer $\mathrm{Y}$, who has a MAP of $p_{1}$ or higher and also a need or strong desire for $2 b_{1}$. Because no larger pack is offered, he will have no option but to buy two of the standard packs, which will cost him $2 p_{1}$.

Suppose now that the vendor withdraws the existing pack 1 and replaces it with a new pack 1 , which contains $2 b_{1}$. Consumer $\mathrm{X}$ will be put in a quandary, but consumer $\mathrm{Y}$, whose MAP is at least $p_{1}$, will be indifferent to this change, and will be content to continue paying $2 p_{1}$ for the same amount of the good, $B$, now contained in the new pack 1 .

Let us now transfer our attention to a person, let us call him consumer $\mathrm{Z}$, with a MAP of $p_{1}$ or more who has a need or strong desire for $4 b_{1}$. Consumer $Z$ would need initially to buy 4 of pack 1 at $p_{1}$ each, then, after the vendor's doubling of the size of pack 1 , would be content to buy 2 of new pack 1 at $2 p_{1}$ each, making his total outlay $4 p_{1}$. Moreover, it would make no difference to him if new pack 1 were supplanted by a further revised pack 1 with contents, $4 b_{1}$, and price $4 p_{1}$. His total bill remains the same in all cases.

Now suppose that the original pack 1 containing $b_{1}$ is reinstated, and moreover a pack 2 containing $2 b_{1}$ is also offered. The pack containing $4 b_{1}$ is retained but it should now be called pack 3 , because there are now two non-empty, smaller packs. The presence of the two smaller packs has given the prospective purchaser more choices, and this makes the pricing of the pack containing $4 b_{1}$ more complicated than it was before. Thus we can no longer say that the price of the pack containing $4 b_{1}$ should be $4 p_{1}$. Nevertheless the price, $4 p_{1}$, retains the significance that it is the price that customer $Z$ would prepared to pay for a pack of size $4 b_{1}$ if no smaller packs were available and the only other option was to buy nothing - the empty pack or pack 0 in our terminalogy. This limiting price for pack 3 may be denoted $p_{30}$, where $p_{30}=L(3) p_{1}=4 p_{1}$.

The larger pack, pack $x$, has not figured in the analysis above, so that its existence or otherwise is not relevant. This will hold true for customers, $\mathrm{X}, \mathrm{Y}$ and $\mathrm{Z}$, as long as the price of pack $x$ is not below that of the largest of the smaller packs. (This can happen on occasion, but rarely on a continuing basis, as it contradicts the economic principle that more will be preferred to less.)

Generalising, we may observe that if a purchaser, say consumer $\Omega$, has a MAP of $p_{1}$ or more for a pack containing $b_{1}$, has a need or strong desire for the quantity contained in pack $n$, namely $L(n) b_{1}$, and he chooses to ignore any packs between pack 0 and pack $n$, then the retailer may continue offering the smaller packs without affecting the valuation, $p_{n 0}$, put on pack $n$ by this purchaser. Because he has decided to ignore smaller packs, person $\Omega$ will be prepared to pay $p_{n 0}$ for pack $n$, given by:

$$
p_{n 0}=L(n) p_{1}
$$

The condition of ignoring packs smaller than pack $n$ will be satisfied automatically when $n=1$, since, by definition, no pack smaller than pack 1 is offered, apart from the empty pack, pack 0 . Thus putting $n=1$ in Equation (3) produces the price for pack 1 :

$$
p_{10}=L(1) p_{1}=p_{1}
$$

Now let us consider utility. The utility resulting from buying pack $n$ will be $u\left(b_{n}\right)$, and the difference, $\Delta u_{n 0}$, between the utility of having purchased pack $n$ and that of having bought pack 0 , i.e. made no purchase, will be given by

$$
\Delta u_{n 0}=u\left(b_{n}\right)-u\left(b_{0}\right)
$$

Assume next that the purchase in question was made on behalf of a consortium of $L(n)$ people, each of whom

- has a MAP of at least $p_{1}$;

- has a need or strong desire for a quantity of the good, $b_{1}$; 
- is allotted $b_{1}$ from the contents, $L(n) b_{1}$, after pack $n$ is purchased.

If we make the assumption that the utility gain for each person in the consortium is the same, then the gain in the utility for each consortium member will be $u\left(b_{1}\right)-u\left(b_{0}\right)$. This will be the same as if that member had himself gone out and bought pack 1, as we may confirm by putting $n=1$ in Equation (5) to give the total utility gain from the purchase of pack 1 as:

$$
\Delta u_{10}=u\left(b_{1}\right)-u\left(b_{0}\right)
$$

The total utility gain for the consortium of $L(n)$ people may be found by adding together all the components identified in Equation (6):

$$
\Delta u_{n 0}=\sum_{i=1}^{L(n)} \Delta u_{10}=\Delta u_{10} \sum_{i=1}^{L(n)} 1=L(n) \Delta u_{10}
$$

demonstrating that the utility gain from pack $n$ will be proportional to the utility gain from pack 1 , with a factor of proportionality, $L(n)$. For the utility gain from pack $n$ to be proportional to the utility gain from pack 1 , then the utility differences for the purchasers of pack $n$ and pack 1 will obey a very restricted version of homogeneity, "singular homogeneity", as explained in Appendix A.

An example of a consortium as described above would be 4 university students sharing a house and joining together to buy a 4-pint pack of milk a day to be divided equally between them. Another consortium could be a mother buying the same pack of milk daily for her family. On the other hand, an individual living alone and consuming milk at the same rate as one of the students might buy a 4-pack of milk every 4 days. In this last case, the consortium consists of the individual on day 1 and the same individual on days 2, 3 and 4. This generalization of the consortium so that it can include time-delayed versions of the same individual makes it reasonable to assume that that every purchaser of pack $n$ experiences the same utility gain, $\Delta u_{n 0}$, whether part of a multi-person consortium or not. This equality in the gain in utility is taken to be independent of the price paid for the pack, and to be valid in cases where the pack-size ratio is noninteger.

Combining Equations (3) and (7), we may write the ratio of the changes in utility in terms of the ratio of the limiting price for pack $n$ to the achieved price for pack 1:

$$
\frac{\Delta u_{n 0}}{\Delta u_{10}}=\frac{p_{n 0}}{p_{1}} \quad 1 \leq n \leq N
$$

But in general there will be packs intermediate between pack $n$ and pack 0 . In this case, when considering the purchase of pack $n$, in addition to the utility difference between pack $n$ and pack 0 , the consumer may well wish to take into account also the utility differences, $\Delta u_{n k}, k<n$, that will arise from comparisons between pack $n$ and packs in between pack $n$ and pack 0 :

$$
\Delta u_{n k}=u\left(b_{n}\right)-u\left(b_{k}\right) \quad 1 \leq k \leq n
$$

The change in utility between buying pack $n$ and making no purchase may be then be expressed in terms of intermediate utility differences:

$$
\begin{aligned}
\Delta u_{n 0} & =u\left(b_{n}\right)-u\left(b_{0}\right)=u\left(b_{n}\right)-u\left(b_{k}\right)+u\left(b_{k}\right)-u\left(b_{0}\right) \\
& =\Delta u_{n k}+\Delta u_{k 0}
\end{aligned}
$$

where the last step has made use of Equation (5), replacing the subscripts, $n$, and 0 with the subscript, $k$, as necessary. Thus the change in utility between purchasing pack $n$ and a smaller pack $k$ may be written in terms of the utility gains from buying pack $n$ and pack $k$, respecttively:

$$
\Delta u_{n k}=\Delta u_{n 0}-\Delta u_{k 0}
$$

Hence

$$
\frac{\Delta u_{n k}}{\Delta u_{k 0}}=\frac{\Delta u_{n 0}}{\Delta u_{k 0}}-1=\frac{\Delta u_{n 0}}{\Delta u_{10}} \frac{\Delta u_{10}}{\Delta u_{k 0}}-1
$$

Substituting from Equation (7) with the appropriate subscripts into Equation (12) gives

$$
\frac{\Delta u_{n k}}{\Delta u_{k 0}}=\frac{L(n)}{L(k)}-1 \quad k>0
$$

Since $L(n)>L(k)$, it follows that $\Delta u_{n k} / \Delta u_{k 0}$ will always be positive.

\section{Comparing Packs of Different Sizes}

Because they are of the same good, packs may be characterised by only two quantities, their size and their price. But while a single comparison involving multiple packs would be feasible if the packs could be characterised by just one quantity, when each pack has even as few as two characteristic features, it becomes difficult if not impossible for the consumer to compare packs more than two at a time, even when both those characteristics are represented numerically. This is exactly the situation addressed by mathematics when comparisons are to be made between an array of properties, formalised mathematically as a vector. Generally it is not meaningful to say that a vector, $\boldsymbol{x}$, is bigger than another, $\boldsymbol{y}$, of the same dimension, the only exception being when the vectors are linearly related: $\boldsymbol{y}=\boldsymbol{\lambda} \boldsymbol{x}$, where $\lambda$ is a constant. In every other case, further mathematical operation is needed before it is possible to make a meaningful comparison, such as taking the modulus: $|\boldsymbol{x}|=\sqrt{\sum_{i} x_{i}^{2}}$, and then comparing the single number $|\boldsymbol{x}|$ with the single number, $|\boldsymbol{y}|$.

When the packs are characterized by size and price, it 
is, of course, possible to divide the price by the size to give the price per unit. This is normally done in UK supermarkets by the vendor, who is obliged to display the result to his customers. But while the prices per unit over all $n$ are easily ordered and hence may be compared multiply, the unit price does not give a full characterization of the pack, for which the size and price are still needed. For example, suppose that the price of the standard pack containing one unit is $£ 2$ and the price of a pack containing 10 units is $£ 19$, then based on the unit price the larger pack is clearly preferable. But the number of units may be more than the purchaser needs or the amount of money required may be more than the consumer wants to lay out on this good, so that actually the consumer will prefer pack 1. Hence the role of the price per unit for pack $n, \pi_{n}$, is to add to the features characterizing the pack, meaning that the vector of characteristics has risen to three:

$$
\left(\begin{array}{c}
L(n) \\
p_{n} \\
\pi_{n}
\end{array}\right)
$$

strengthening the necessity for pair-wise comparisons.

Consumers may carry out comparisons over the whole range of packs, or they may make an outline decision early on in the selection process on the range of packs that they judge may meet their desire for the good, $B$, and their budgetary limitations. In each case, the pair-wise comparison will be made by taking each pack, pack $n$, within the feasible range, and comparing it with a smaller pack, $k: 0<k<n$. A price, $p_{n k}$, will emerge from each comparison. The consumer is presumed eventually to eliminate from consideration all sizes greater than his ideal size, and base the price he is prepared to pay for this ideal size on the outcome of some or all of the comparisons he has made using this pack as his basis. Thus the final price for pack $n$ will be a function of the comparison-generated prices or a subset of them:

$$
p_{n}=g\left(p_{n 0}, p_{n 1}, \cdots, p_{n k}, \cdots, p_{n, n-1}\right)
$$

Thus the set of feasible binary comparisons for pack $n$ will be between pack $n$ and the following packs, taken one at a time:

- $\operatorname{pack} 0$ (= the empty pack or no purchase).

- pack 1 .

- $\quad \vdots$

- pack $k$.

- $\quad \vdots$

- pack $n-1$.

At each comparison between pack $n$ and pack $k$, the consumer is presumed to ask himself the question:

"Suppose I am on the point of agreeing to buy pack $k$, what will it take to make me want to buy the greater quantity contained in pack $n$ ?"

He may then reason that the best answer to this question will be found by consulting a mentor with greater relevant experience, namely someone who has actually bought pack $k$. This strategy may be characterised as "phone a friend". The mentor has something the consumer does not: he knows what it feels like actually to own pack $k$. He has knowledge borne of experience, the knowledge of possession.

Let us now consider the perspective of the mentor. As a purchaser of pack $k$, the mentor may be presumed to have most of his need satisfied by pack $k$, which contains $L(k)$ units. Buying pack $n$ in addition will give him $L(n)+L(k)$ units. But $L(n)$ will be significantly greater than his original target, $L(k)$, and so those $L(n)$ units may be presumed to meet his wants in full. Hence $L(k)$ units out of the $L(n)+L(k)$ will now be surplus to requirement, and bring the mentor no utility. As a result, after purchasing packs $k$ and $n$, the person who originally bought pack $k$ will take the utility gain from pack $k$ and $n$ together as equal simply to the utility gain from pack $n$. So in considering whether to buy pack $n$, given that he possesses pack $k$, the mentor will be prepared to pay for only the extra utility that pack $n$ adds over and above that provided by pack $k$, that is to say, $\Delta u_{n k}$. We may presume that he would advise the prospective purchaser accordingly.

We may further suppose that the prospective purchaser of pack $n$ making the comparison with pack $k$ will accept the advice of his $k-n$ mentor and take the utility gain from pack $n$ as relative to the gain brought by pack $k$, namely, $\Delta u_{n k}$. By Equation (11), $\Delta u_{n k}$ is the margin between the utility gain from of pack $n$ and the utility gained from pack $k$, and this is smaller than the utility gain, $\Delta u_{n 0}$. Thus, when the needs or desires of a prospective purchaser of a commodity are close to being satisfied by a pack smaller than pack $n$, that purchaser will see the acquisition of pack $n$ bringing him less than its full utility.

It will be apparent to the reader at this point that the prospective purchaser does not actually need to "phone a friend" at all. He can work it out for himself simply by imagining how he would feel if he had, in fact, bought pack $k$.

Such an approach is likely to work well with commodities, which are by their nature simple. The demands on the consumer's imagination might become more taxing with more complex and differentiated goods, where real, third-party advice might be needed. But such differentiated goods tend to be sold as one-offs rather than in packs, taking away the need for a $k-n$ comparison. The vendor may, of course, attempt to provide differentiation for goods that many would regard as commodities, and a discussion of such situations is given as part of the field studies included in [13]. 


\section{Prices Arising from a Binary Comparison between Pack $n$ and Pack 1}

If the consumer narrows his final pair-wise comparison down to either buying pack $n$ or making no purchase (buying pack 0 ), the price he will be prepared to pay will be $p_{n 0}$, as given by Equation (3). This represents the case of a consumer with a MAP of at least $p_{1}$ who considers that he has such a strong need for $L(n)$ standard units that he is not prepared to take any lesser quantity and decides to ignore all packs of intermediate sizes. It is this that causes him to value those $L(n)$ units on the full utility gain rather than their utility relative to some intermediate purchase.

It may be, however, that the consumer will grant himself the flexibility to make a comparison between pack $n$ and the first pack. Let the price that he is prepared to pay for pack $n$ when his comparison is between pack $n$ and pack 1 be $p_{n 1}$. We now assume that the price per unit gain in utility stays constant for all transactions he is considering - a standard economic assumption, e.g. [14]. Hence

$$
\frac{p_{n 1}}{\Delta u_{n 1}}=\frac{p_{1}}{\Delta u_{10}}
$$

Meanwhile the ratio of utility differences, $\Delta u_{n 1} / \Delta u_{10}$, may be found by putting $k=1$ in Equation (13):

$$
\frac{\Delta u_{n 1}}{\Delta u_{10}}=L(n)-1
$$

Since $L(1)=1$, the price that the consumer will be prepared to pay for pack $n$, if his comparison narrows down finally to the purchase of pack $n$ or pack 1 , may be found by combining Equations (15) and (16) and also using $p_{10}=p_{1}$ from Equation (4):

$$
p_{n 1}=\frac{\Delta u_{n 1}}{\Delta u_{10}} p_{1}=\max (1.0, L(n)-1) p_{1}
$$

A maximum function has been added in Equation (17), embodying the general economic principle that, all other things being equal, less will not be preferred to more, and so the larger pack will not attract a lower price. (Without it, a largeness of $L(n)=1.5$, for example, would imply that the price of pack $n$ would be lower than that of the smaller pack 1 . While such a pricing tactic is not unknown as a marketing promotion, its use is rarely maintained in the long term, as noted previously.)

Noting that $L(n)>1$, it will be seen by comparing Equations (3) and (17) that the price derived by compareing pack $n$ with pack 1 cannot be greater than the price achieved by comparing pack $n$ with pack 0 . Since some consumers of pack $n$ are almost certain to make the former comparison, the retailer will need to accommodate the likely lower valuation to some degree, and will set a price for pack $n$ that is lower than would be implied by proportionality to size. In general, the achieved price, $p_{n}$, for any pack, $n: n>1$, is almost certain to be less than $p_{n 0}$ :

$$
p_{n} \leq p_{n 0}
$$

This provides the theoretical basis for quantity discounts.

\section{Prices Arising from a General Binary Comparison between Pack $n$ and Pack $k$; the Single Comparison Relative Utility Pricing Model (SCRUP)}

When the purchase of pack $n$ is being considered relative to the purchase of pack $k$ rather than pack 1 the assumption of constant price per unit utility gained produces Equation (19), which is analogous to Equation (15):

$$
\frac{p_{n k}}{\Delta u_{n k}}=\frac{p_{k}}{\Delta u_{k 0}}
$$

where $p_{k}$ is the achieved price for pack $k$. As noted previously in Section 3, the same gain in utility, $\Delta u_{k 0}$, is assumed for all purchasers of pack $k$, as found from Equation (5) after substituting $k$ for $n$.

Combining Equations (13) and (19) gives the price for pack $n$ emerging from the comparison of pack $n$ and pack $k$ as:

$$
p_{n k}=\max \left(1.0, \frac{L(n)}{L(k)}-1\right) p_{k} \quad k \geq 1
$$

Once again, the maximum function embodies the general economic principle that, all other things being equal, less will not be preferred to more, and so the greater quantity will not attract a lower price. Equations (20) and (3) together give $p_{n k}$ for all $k \geq 0$, and, of course, Equation (20) takes the form of Equation (17) when $k=$ 1.

The customer considering buying pack $n$, of largeness, $L(n)$, will have the following possible prices available to him, based on his comparisons with other, smaller packs, ranging from the empty pack, pack 0 , to the pack immediately below pack $n$ in size, namely pack $n-1$ :

$$
p_{n 0}, p_{n 1}, \cdots, p_{n k}, \cdots, p_{n, n-1}
$$

The good in pack $n$ is effectively competing with itself contained in smaller packs. The maximum acceptable price (MAP) for individual, $i$, interested in purchasing pack $n, p_{n}^{(i)}$, will therefore be a function of the prices shown in list (21). It is suggested that the dependency can be represented by the weighting function:

$$
\begin{aligned}
p_{n}^{(i)} & =w_{0}^{(i)} p_{n 0}+w_{1}^{(i)} p_{n 1}+\cdots+w_{k}^{(i)} p_{n k}+\cdots+w_{n-1}^{(i)} p_{n, n-1} \\
& =\sum_{k=0}^{n-1} w_{k}^{(i)} p_{n k}
\end{aligned}
$$


where $w_{k}^{(i)}, k=0,1, \cdots, n-1$ are a series of weighting factors particular to the individual, which will be subject to the conditions:

$$
\begin{aligned}
& 0 \leq w_{k}^{(i)} \leq 1 \\
& \sum_{k=0}^{n-1} w_{k}^{(i)}=1
\end{aligned}
$$

The aggregation of these MAPs will produce a probability density for MAP for the target population, which will be constrained to the interval, $\left(p_{n-1}, L(n) p_{1}\right)$, as shown in Appendix B.

In considering whether or not to purchase pack $n$, of a commodity, the greatest weight will usually be given to the comparison between pack $n$ and the pack just below it in size, pack $n-1$. In the limiting case where this comparison is completely dominant, $w_{n-1}^{(i)}=1$.

All other comparisons are now either not made or are considered irrelevant. The achieved price for pack $n$ will be $p_{n}=p_{n, n-1}$ when all the prospective purchasers adopt this policy. We may describe this as the Single Comparison Relative Utility Pricing (SCRUP) model, which will be shown to have the power to explain a number of everyday pricing strategies the economics of which have been understood only poorly until now.

\section{Using the SCRUP Model to Explain the BOGOF and 3-for-the-Price-of-2 Promotions}

To illustrate the results and the power of the SCRUP model, we shall consider first the case of the pricing of pack 2, when there is only one pricing comparison that can be made, that is to say between pack 2 and pack 1 . Here $n=2$ and $k=1$. Let us consider first the case where pack 2 holds twice the contents of pack 1 so that $L(2)=2$. If buyers choose to compare the two packs, we have, after substituting the appropriate values into Equation (20):

$$
p_{21}=\max (1.0,2-1) p_{1}=p_{1}
$$

This means that if the purchasers of a commodity have needs or desires that are close to being satisfied by pack 1 and so are making a serious comparison between pack 1 and a pack 2 that is twice the size, then they will be prepared to pay only the same for the larger pack as they were prepared to pay for the smaller pack. Prima facie surprising, this result nevertheless provides a complete explanation for why the vendor needs to offer the apparently generous BOGOF promotion.

By a combination of technological convenience and custom and practice, universal serial bus (USB) memory sticks are offered with storage capacities ascending in multiples of 2. The lack of novelty associated with the lower-capacity sticks means that they are regarded purely as commodity item. Hence, by Equation (24), we can expect the price of the two lowest-capacity sticks to be equal or nearly so. Hence in December 2009, the entry-level $1 \mathrm{~Gb}$ USB memory stick was on sale on the internet at $£ 6.96$, while a $2 \mathrm{~Gb}$ stick from the same manufacturer was offered at a comparable price - in fact slightly lower at $£ 6.44$ [15]. This pricing was not a one-off, as demonstrated by the pricing, early in August 2013, of the entry-level pack, now containing $4 \mathrm{~Gb}$ of memory at $£ 5.79$, while the next pack up from the same manufacturer, containing $8 \mathrm{~Gb}$, was on sale for almost the same price, $£ 6.03$. The pricing of such memory sticks is considered in more detail in [13].

The SCRUP model can also explain immediately a perplexing instance of everyday pricing. It used to be normal practice in the UK to sell eggs by the dozen, but supermarkets have taken to making half a dozen the size of pack 1 for "value" eggs. Surprisingly, UK supermarkets no longer sell "value" eggs in packs of a dozen [16].

But if they did, Equation (24) shows that they would find themselves compelled to sell both 6 egg and 12 egg packs at the same price, a situation with which they would probably not be happy on a continuing basis. Instead major UK supermarkets have taken to selling their "value" eggs with a pack 1 set at 6 eggs and a pack 2 containing either 15 eggs or 18 eggs.

Considering the case of packs of "value" eggs of 6 and 18, substituting, $n=1, k=1$ and $L(2)=3$ into Equation (20) produces:

$$
p_{21}=\max (1.0,3-1) p_{1}=2 p_{1}
$$

The achieved price predicted by the SCRUP model for pack 2 will be equal to the result of Equation (25), viz. $p_{2}=p_{21}=2 p_{1}$. Equation (25) provides the explanation for the other common sales promotion: 3-for-the-priceof-2.

Validation for this prediction of the SCRUP model comes from the prices charged by one of the UK's largest supermarket chains, namely Sainsbury. In that supermarket, a pack of 6 "Basics Barn Eggs" (pack 1) was on sale for $£ 0.91$ in December 2009, while pack 2, which contained 18 eggs, retailed for $£ 1.85$ [16]. This is within $2 \%$ of the theoretical figure from Equation (25) of $2 \times £ 0.91=£ 1.82$. Further discussion of prices achieved will be given in Section 8.3 and, in greater detail, in [13].

An example of a quantity offer that the SCRUP model makes no claim to explain is the promotion, "Buy one get one half price". How is it that the retailer is able to command $1 \frac{1}{2}$ times the price for two items, rather than just the same price for two items as for one item? In fact, this promotion is commonly used in selling paperback books, 
for example by the large U.K. retailer, W. H. Smith. W. H. Smith applies this offer both to fiction and to paperbacks on management, economics, business and self-help, or to any mixture of the categories. But given that the customer is unlikely to buy two of the same book, the offer may be seen to concern two complex and differenttiated goods, which do not fall within the capability of the SCRUP model, as explained at the end of Section 4. The interesting feature here is that the retailer has decided to increase his sales of paperback books by regarding these items as half-way to being commodities, and this allows him to use a variant of the promotional techniques that apply to retail commodities.

\section{Comparing the Price per Unit from the SCRUP Model with the Price per Unit from the Iso-Elastic Utility Function}

In this Section we will restrict our attention to the common case where the packs increase in size by a constant pack size ratio, $\rho$.

\subsection{Price per Unit from the SCRUP Model}

In this analysis we will drop the maximum function from Equation (20), which may therefore be written in the simpler form:

$$
p_{n k}=(L(n)-L(k)) \frac{p_{k}}{L(k)} \quad k \geq 1
$$

Equations (20) and (26) will give identical results when $\rho=L(n) / L(n-1) \geq 2$, and dropping the restriction of the maximum function will allow the exploration of inelastic demand.

The achieved price predicted by the SCRUP model will be $p_{n, n-1}$, allowing us to write $p_{n}=p_{n, n-1}$. Substituting $n-1$ for $k$ and dividing both sides of Equation (26) by the number of units in pack $n, L(n)$, gives:

$$
\frac{p_{n}}{L(n)}=\left(1-\frac{L(n-1)}{L(n)}\right) \frac{p_{n-1}}{L(n-1)} \quad n \geq 2
$$

The price per unit for pack $n, \pi_{n}$, is:

$$
\pi_{n}=\frac{p_{n}}{L(n)}
$$

and so Equation (27) may be rewritten:

$$
\frac{\pi_{n}}{\pi_{n-1}}=1-\frac{L(n-1)}{L(n)}=1-\frac{1}{\rho}=\frac{\rho-1}{\rho} \quad n \geq 2
$$

Prices per unit relative to the price per unit for pack 1, $\pi_{n} / \pi_{1}$ may be found successively from:

$$
\begin{aligned}
& \frac{\pi_{2}}{\pi_{1}}=\frac{\rho-1}{\rho} \\
& \frac{\pi_{3}}{\pi_{1}}=\frac{\pi_{2}}{\pi_{1}} \frac{\pi_{3}}{\pi_{2}}=\left(\frac{\rho-1}{\rho}\right)^{2}
\end{aligned}
$$

and so on, so that the general expression is

$$
\frac{\pi_{n}}{\pi_{1}}=\left(\frac{\rho-1}{\rho}\right)^{n-1} \quad n \geq 1
$$

These per unit price ratios will be valid at the discrete points corresponding to the various pack sizes.

\subsection{The Price per Unit from the Iso-Elastic Utility Function}

If we assume that the amount of good, $B$, that may be contained in a pack is a continuous variable, $b$, the gain in utility may be modelled by the continuous utility function, $u(b)$. The price per unit or price density will now also be continuous in $b: \pi(b)$. Hence the cost of buying an extra, small amount, $\delta b$, starting from an initial amount, $b$, will be $\pi(b) \delta b$. In the case where the quantity is equal to the amount contained in the starting pack, pack 1 , then $b=b_{1}$, and the cost of a further small amount, $\delta b$, will be approximately $\pi\left(b_{1}\right) \delta b$.

Meanwhile, the extra utility associated with having acquired an extra, small amount, $\delta b$, of $B$ in addition to amount, $b$, will be

$$
\delta u(b)=u(b+\delta b)-u(b)
$$

Correspondingly, the extra utility associated with a small amount, $\delta b$, of good, $B$, added to $b_{1}$, the contents of pack 1 , will be

$$
\delta u\left(b_{1}\right)=u\left(b_{1}+\delta b\right)-u\left(b_{1}\right)
$$

The standard relationship between price and marginal utility used in Equations (15) and (19) may be extended to the case of a continuous quantity, $b$, so that the ratio of Equations (33) and (32) gives:

$$
\frac{\pi(b) \delta b}{\pi\left(b_{1}\right) \delta b}=\frac{\delta u(b)}{\delta u\left(b_{1}\right)}
$$

or

$$
\frac{\pi(b)}{\pi\left(b_{1}\right)}=\frac{\delta u(b)}{\delta b} \div \frac{\delta u\left(b_{1}\right)}{\delta b}=\frac{u^{\prime}(b)}{u^{\prime}\left(b_{1}\right)} \quad \text { as } \delta b \rightarrow 0
$$

An iso-elastic utility function, $u(b)$, giving the utility of a continuous quantity, $b$, has the property that the elasticity of marginal utility is constant, specifically independent of $b$. Pratt [17] and Arrow [18] showed that the negative of the elasticity of marginal utility was equal to the coefficient of relative risk aversion, $\varepsilon$, which we shall denote "risk-aversion" for short. Thus 


$$
\varepsilon=-\frac{\mathrm{d}(\mathrm{d} u(b) / \mathrm{d} b)}{\mathrm{d} b} \div \frac{\mathrm{d} u(b) / \mathrm{d} b}{b}=-b \frac{u^{\prime \prime}(b)}{u^{\prime}(b)}
$$

For an iso-elastic utility function to be smooth and regular in risk-aversion, it must take the form [19]:

$$
u(b)= \begin{cases}\frac{b^{1-\varepsilon}-1}{1-\varepsilon} & \text { for } \varepsilon \neq 1 \\ \ln b & \text { for } \varepsilon=1\end{cases}
$$

This has the derivative with respect to $b$ :

$$
u^{\prime}(b)=\frac{1}{b^{\varepsilon}} \quad \text { for all } \varepsilon
$$

Combining Equations (35) and (38) gives:

$$
\frac{\pi(b)}{\pi\left(b_{1}\right)}=\frac{b_{1}^{\varepsilon}}{b^{\varepsilon}}
$$

If we convert $b$ into standard units, with 1 standard unit corresponding to the contents of pack $1: b_{1}=1$, then:

$$
\frac{\pi(b)}{\pi(1)}=\frac{1}{b^{\varepsilon}}
$$

The elasticity of demand with respect to price or "demand elasticity", $\eta$, is the normalised change in quantity sold with price, given by

$$
\eta=\frac{\mathrm{d} b}{\mathrm{~d} \pi} \div \frac{b}{\pi}
$$

Applying Equation (41) to Equation (40) shows that the demand elasticity, $\eta$, is the negative inverse of the risk-aversion:

$$
\eta=\frac{\mathrm{d} b}{\mathrm{~d} \pi} \div \frac{b}{\pi}=-\frac{1}{\varepsilon}
$$

When risk-aversion, $\varepsilon=1$, the demand elasticity will take the neutral value, $\eta=-1$, neither elastic nor inelastic. Furthermore, inspection of Equation (40) shows that the locus of $\pi(b) / \pi(1)$ vs. $b$ will be a rectangular hyperbola when $\varepsilon=1$.

Since all packs will be at least as big as the standard, entry-level pack, we need consider only that part of the curve where $b \geq 1$. For this sector of the graph, the price-ratio curve will lie above the rectangular hyperbola when the risk-aversion, $\varepsilon$, conforms to $\varepsilon<1$, which means that the demand elasticity, $\eta<-1$, and the demand is elastic. The price-ratio curve will lie below the hyperbola when the risk-aversion obeys $\varepsilon>1$, signifying a demand elasticity, $\eta>-1$, implying inelastic demand.

The contents of pack $n$ will be $\rho^{n-1} b_{1}$. Hence, noting also that $b$ is now denominated in standard units, so that $b_{1}=1$, we may set $b$ to:

$$
b=\rho^{n-1}
$$

The price per unit required to clear $b=\rho^{n-1}$ units of the continuous good will be given by substituting Equation (43) into Equation (40)

$$
\frac{\pi\left(\rho^{n-1}\right)}{\pi(1)}=\frac{1}{\rho^{\varepsilon(n-1)}}
$$

\subsection{Equating Prices from the SCRUP and Utility Models}

Equating the prices required to clear $b=\rho^{n-1}$ units of the continuous good with the price the SCRUP model suggests for a pack containing $\rho^{n-1}$ units means that we may equate the right-hand sides of Equations (31) and (44):

$$
\left(\frac{\rho-1}{\rho}\right)^{n-1}=\left(\frac{1}{\rho^{\varepsilon}}\right)^{n-1}
$$

which gives the relationship between risk-aversion, $\varepsilon$, and pack size ratio, $\rho$, as:

$$
\varepsilon=1-\frac{\ln (\rho-1)}{\ln \rho}
$$

Equation (46) shows immediately that a continual doubling of the pack size, viz. $\rho=2$, is consistent with a risk-aversion, $\varepsilon=1$, which will give rise to a logarithmmic utility function, as recommended by H. M. Treasury for the evaluation of public-service schemes [20]. As demonstrated above, this produces the neutral demand elasticity, $\eta=-1$. Figure 1 shows the plot of riskaversion versus pack-size ratio.

Meanwhile a risk-aversion, $\varepsilon=0$, may be seen to require an infinite pack size ratio: $\rho=\infty$. This may be deduced from Figure 1 or directly from Equation (46) and verified intuitively by the following argument. The impossibility of constructing an infinitely large pack corresponding to $\rho=\infty$ means that pack 1 will be the

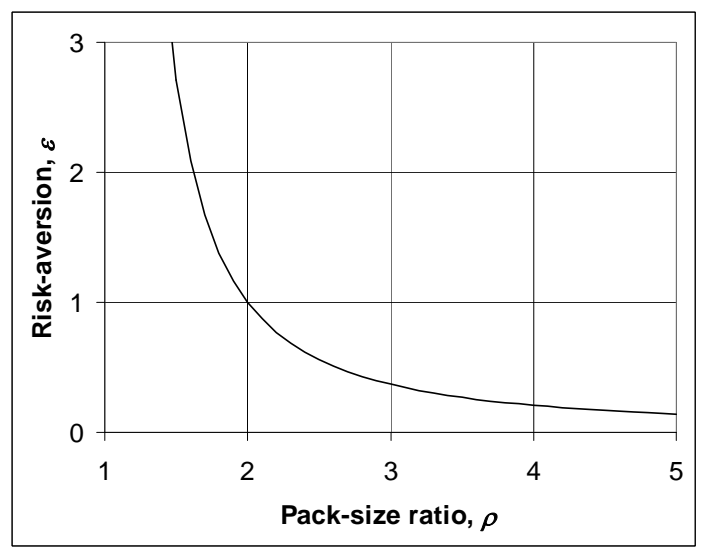

Figure 1. Risk-aversion versus pack-size ratio. 
only pack. Hence all quantities will be supplied as multiplies of pack 1, implying that the per-unit price will stay constant. By Equation (35), an unvarying per-unit price means that the slope of the utility function will be constant so that the utility function must be linear. By inspection, the iso-elastic utility function of Equation (37) becomes linear in $b$ only for $\varepsilon=0$.

Equation (46) will not predict a risk-aversion with a negative value, $\varepsilon<0$. This means that the analysis in this Section will include only those who see either constant or diminishing returns from extra amounts of the good, $B$, and will preclude those who see increasing returns from extra amounts of the good, $B$. This does not constitute a significant practical limitation, since it is assumed generally in economics that the individual will perceive diminishing marginal utility from larger amounts.

Amongst those with positive risk-aversions, who therefore will see strictly diminishing returns from extra amounts of the good, $B$, let us define as "cautious" those with risk-aversions in the range, $0<\varepsilon \leq 1$. Cautious individuals will always perceive a benefit from more of the good, even when the quantity is very high, since their utility, $u(b)$, will increase without limit as $b$ increases. By contrast, let us call those with a risk-aversion strictly greater than unity, $\varepsilon>1$, "ultra-cautious". The utility, $u(b)$, of ultra-cautious individuals will approach an upper, asymptotic limit as $b \rightarrow \infty$. Moreover, it is clear from Figure 1 that a risk-aversion of greater than unity will call for a pack-size ratio of less than 2 . Therefore the limit contained in Equation (17) will always need to be invoked, and even then such ultra-cautious individuals will want to pay the same price for all packs, irrespective of size.

While the vendor may face a market composed of cautious and ultra-cautious individuals, only the cautious consumers will always want more and only the cautious, not the ultra-cautious, may be prepared to pay more for a larger pack. Therefore it is only the cautious consumers whom it is sensible for the vendors to target with quantity promotions.

In the absence of more precise information, it is reasonable to assume a uniform probability distribution for risk-aversion amongst the cautious consumers, over the open interval, $0<\varepsilon \leq 1$. The average value of this distribution is $\varepsilon=0.5$, and, indeed, the same mean will result from any symmetrical distribution over the same interval. Thus, in the absence of evidence to the contrary, the figure, $\varepsilon=0.5$, may be regarded as the risk-aversion for the average person likely to be interested in a sales promotion offering greater quantities. This value of risk-aversion requires from Equation (46) that

$$
\rho=\phi^{2}=\phi+1
$$

where $\phi$ is the golden ratio (see e.g. [21]). (The result is easily confirmed from Equation (46) by using Equation (47) to replace $\ln (\rho-1)$ with $\ln \phi$ and substituting $\ln \rho=\ln \phi^{2}=2 \ln \phi$. Hence $\varepsilon=1-\ln \phi /(2 \ln \phi)=0.5$.) The properties of $\phi$ mean that when the pack size ratio is set to its square, $\phi^{2}$, then the normalised difference in size between two successive packs will be equal to the golden ratio:

$$
\frac{L(n)-L(n-1)}{L(n-1)}=\phi
$$

We may combine Equations (27) and (47) to show that the ratio of the price of pack $n$ to the price of pack $n-1$ will also be equal to the golden ratio:

$$
\frac{p_{n}}{p_{n-1}}=\phi
$$

The value of $\phi$, found from solving the quadratic equation of Equation (47), is approximately 1.62, so that, from the same equation, $\phi^{2} \approx 2.62$.

On this basis, if the vendor is to increase pack sizes by a constant ratio, $\rho$, within a range of quantities known to be desired by cohorts of his customers, the vendor can expect to be match his market most accurately if he sets the size ratio to the square of the Golden Ratio (Equation (47)) and his price ratios at the Golden Ratio (Equation (49)). This result will clearly apply to the case where just two packs are offered, when the size ratio of pack 2 to pack 1 should be the square of the golden ratio and the price ratio should equal the golden ratio. The matching of the pack size and its prices to the values desired by the average customer liable to be tempted by a multibuy offer means that there is a strong case for regarding Golden Ratio sizing and pricing as delivering what the customer wants most.

Figure 2 gives the normalised price per standard unit for packs of different sizes when the comparison is always made with the pack immediately below (the SCRUP model), and when the pack-size ratio, $\rho$, stays constant. The Figure illustrates the cases where this ratio takes the values, 2, 2.5, $\phi^{2}$ and 3, and shows how the locus derived from iso-elastic utility functions with corresponding risk-aversions $1.0,0.56,0.5$ and 0.37 respectively will intersect the discrete data points. Also plotted is the locus corresponding to a pack size ratio of 50: the risk-aversion, at 0.005 , is now close to zero, implying an absolutely very large demand elasticity of -200 , and a price density vs. quantity curve that is nearly horizontal.

Figure 2 demonstrates that when the pack-size ratio is 2, the SCRUP Model will predict a price equal to that produced by a logarithmic utility function. This corresponds to the case where a pack containing double the quantity will attract the same price as the first pack, as 
may be seen from Equation (24). The demand elasticity is neutral under this condition. The retailer may move the market into a region of elastic demand by using a higher pack-size ratio, and can expect to achieve better per unit prices as a result. However, as illustrated by Figure 2 and as a moment's thought will confirm, these higher per unit prices will come at the expense of poorer coverage of the range of potential purchases. While higher size ratios (e.g. a ratio of 3) should give higher per unit prices, the square of the golden ratio $(\sim 2.62)$ should offer an optimal compromise between higher per unit price and coverage of the range of quantities required by consumers.

More detail on the prices achieved by major UK supermarkets will be given in [13]. Here we will content ourselves with a brief discussion of one result given there, concerning "value" eggs where the size of pack 1 was 6 eggs and the size of pack 2 was either 18 eggs (Sainsbury) or 15 eggs (Asda and Tesco) and where pack 1 was retailing at the same price, $£ 0.91$, in all three supermarkets. The price per egg achieved by Sainsbury for its 18-egg pack 2 was $£ 1.85 / 18=£ 0.103$, which is within $2 \%$ of the theoretical figure of $(18 / 6-1) \times £ 0.91 / 18=£ 0.101$.

As noted in the previous paragraph, pack 2 for value eggs in Asda and Tesco contained 15 eggs, giving a pack-size ratio of 2.5 , which is significantly closer to the ideal ratio derived above of $\phi^{2}=2.62$. By selling pack 2 at $£ 1.50$, these stores achieved a price per egg of $£ 0.100$ with their pack 2 , which is $9 \%$ higher than the predicted price of $(15 / 6 \times £ 0.91) / 15=£ 0.091$. The ideal number of eggs in pack 2 based on this theory would be $\phi^{2} \times 6=15.72$ or 16 after rounding. A pack 2 containing 16 eggs should command a price of

$(16 / 6-1) \times £ 0.91=£ 1.52$, giving a price per egg of $£ 0.095$. The premium price per egg achieved by Tesco and Asda with their pack 2 may result from the pack size

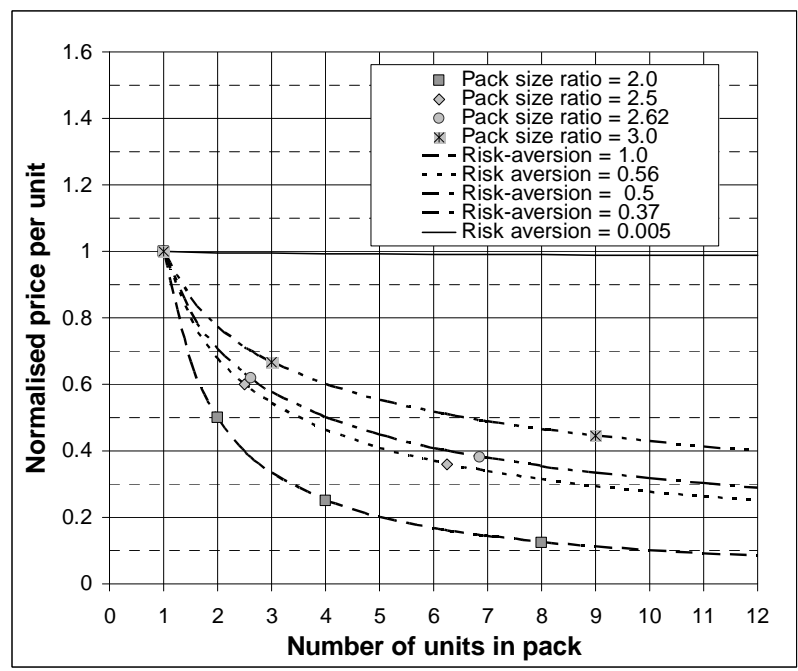

Figure 2. Effect of pack size ratio on the price per unit. satisfying better people's sense of the most appropriate next size up. On the other hand, it may well be that they priced their pack 2 based on the price per egg that their competitor, Sainsbury, was achieving, and might have done better through higher sales by pricing their pack 2 at $(15 / 6-1) \times £ 0.91=£ 1.37$. The theory just developed suggests that they might have done better still by putting 16 eggs into their pack 2, which they would then have sold at $£ 1.52$, since this would have matched the requirements of the average cautious customer best.

Interestingly, by August 2013, Tesco was pricing its value eggs at $£ 0.87$ for a half dozen and $£ 1.35$ for 15 [13]. Using the SCRUP model, the predicted price for 15 eggs would be $(15 / 6-1) \times 0.87=£ 1.31$. The actual price had thus drawn significantly closer to the predicted value - a $3 \%$ difference as opposed to the earlier $9 \%$ difference.

\section{The Retailer's Problem: Choosing the Largeness of Pack 2 to Maximise Profit: BOGOF vs. 3-for-2 vs. Golden Ratio Pricing}

Fundamentally, although the customer will want quantity discounts, the retailer will be prepared to offer them if his variable costs are low enough for each sale to make a non-negative contribution to covering his overall costs. The purpose of this Section is to examine the circumstances when the vendor can maximise his profit by setting the largeness of pack 2 as $L(2)=2$, as in a BOGOF, when it is best to set it as $L(2)=3$, as in a 3 -for-2 offer, and when the most profitable strategy might be to set the largeness of pack 2 at the square of the Golden Ratio, $L(2)=\phi^{2} \approx 2.62$. This Section is therefore concerned with the overall design of pack 2 , in terms of both size and price.

\subsection{The Optimising Equation}

The exercise of choosing the largeness of pack 2 to maximise the vendor's profits may be treated in a similar way to that presented in [11] by using a probability distribution to model the maximum acceptable price (MAP) amongst those making up the target market. The further complication in this case is that the MAP for pack 2 varies with its largeness. For the cases that we want to consider, where the pack-size ratio is at least two: $\rho \geq 2$, the relationship is given by putting $n=2$ into Equation (27) and rearranging:

$$
p_{2}=\left(\frac{L(2)}{L(1)}-1\right) p_{1}=(\rho-1) p_{1} \quad \rho \geq 2
$$

where, since $L(1)=1$, the largeness of pack 2 is identical with the pack-size ratio, $L(2)=\rho$. For convenience, let us define the price of pack 1 as our base money unit 
so that $p_{1}=1$ and the price of pack $2, p_{2}$, is expressed as a multiple of the price of pack 1 . Using this system of units, we may then simplify Equation (50) into the form:

$$
p_{2}=\rho-1 \quad \rho \geq 2
$$

The variable cost of pack $1, c_{v}$, may be expressed in terms of the same base unit, the price of pack 1. Assuming moreover that the income from selling pack 1 will cover at least its variable cost, $c_{v}$ will lie in the defined range:

$$
0 \leq c_{v} \leq 1.0
$$

Since pack 2 contains a factor, $\rho$, times the contents of pack 1, then the variable cost associated with pack 2 will be $\rho c_{v}$, or, using Equation (51), $c_{v}\left(p_{2}+1\right)$.

Let the probability density for the MAP for pack 2, $p_{2}$, be $h\left(p_{2}\right)$. Using the concept of the "uniconsumer" introduced without loss of generality in [11] to characterise a person prepared to buy one but only one item if the price is right, the fraction of the uniconsumers prepared to pay $p_{2}$ or more for a single pack 2 will be $S\left(p_{2}\right)$, given by

$$
S\left(p_{2}\right)=\int_{p_{2}}^{p_{2} m} h(v) \mathrm{d} v=1-\int_{0}^{p_{2}} h(v) \mathrm{d} v
$$

where $p_{2 m}$ is the most that anyone in the target market is prepared to pay for pack 2 . Those prepared to pay more would ideally want a larger pack 2 for their money, but it is assumed that they would be content with the smaller pack 2, provided it cost less, if that was all that was offered.

Let there be $N$ uniconsumers in the target group. The vendor's total profit, $\Psi$, from selling packs 2 will be his total income for pack 2 less both the total variable costs and the fixed costs associated with pack $2, C_{F}$ :

$$
\Psi=N S\left(p_{2}\right) p_{2}-N S\left(p_{2}\right) c_{v}\left(p_{2}+1\right)-C_{F}
$$

The retailer will seek to maximise this profit, which, for a constant size of target population, $N$, is equivalent to maximising the average profit per consumer, $\psi$ :

$$
\psi=\frac{\Psi}{N}=\left(p_{2}\left(1-c_{v}\right)-c_{v}\right)\left(1-\int_{0}^{p_{2}} h(v) \mathrm{d} v\right)-\frac{C_{F}}{N}
$$

where use has been made of Equation (53) in the second step.

The maximum value of profit, $\psi$, may be found by differentiating Equation (55) with respect to pack 2 price, $p_{2}$, and then setting $\mathrm{d} \psi / \mathrm{d} p_{2}=0$. This gives the optimal price as the solution, $p_{2}$, of

$$
\left(1-c_{v}\right)\left(1-\int_{0}^{p_{2}} h(v) \mathrm{d} v\right)-\left(p_{2}\left(1-c_{v}\right)-c_{v}\right) h\left(p_{2}\right)=0
$$

which will be seen to depend on the variable cost associ- ated with pack $1, c_{v}$, and the probability distribution, $h\left(p_{2}\right)$, for pack 2 price, $p_{2}$.

A limit in the range of risk-aversions is implicit in the assignment of the maximum price that anyone would pay for a pack 2, $p_{2 m}$, in Equation (53), and it is appropriate now to consider the basis for this limit. The maximum price, $p_{2 m}$, that anyone is prepared to pay for pack 2 , will impact on the maximum largeness, $\left.L(2)\right|_{\max }$, via Equation (51): $p_{2 m}=\rho_{m}-1$ where $\rho_{m}$ is the maximum pack-size ratio and equal to the maximum largeness for pack 2: $\rho_{m}=\left.L(2)\right|_{\max }$. The maximum pack -size ratio, $\rho_{m}$, will imply a lower limit on the risk-aversion via equation (46):

$$
\varepsilon_{m}=1-\frac{\ln \left(\rho_{m}-1\right)}{\ln \rho_{m}}
$$

When he is considering assigning a largeness for pack 2 of between 2 and 3, we can expect that the vendor will have an idea of the maximum size for pack 2 wanted by even the consumers possessed of the largest appetite for that good. The lowest maximum largeness we can take to be 3 (otherwise why would he be considering this as a possible size for pack 2?), and twice that figure, namely 6 , would seem to constitute a reasonable estimate of the highest maximum largeness in the absence of more precise information. Putting $\rho_{m}=3$ into Equation (57) gives a minimum risk-aversion of $\varepsilon_{m}=0.369$, while inserting $\rho_{m}=6$ gives $\varepsilon_{m}=0.101$. In the latter case, about $90 \%$ of the possible range of risk-aversion for cautious consumers is covered: $0.101 \leq \varepsilon_{m} \leq 1.0$.

\subsection{Distributions for Risk-Aversion}

The distribution of risk-aversion has been modelled in the first instance as a uniform distribution over the restricted range: $\varepsilon_{m} \leq \varepsilon \leq 1.0$, so that the probability density, $g(\varepsilon)$ for risk-aversion, $\varepsilon$, is:

$$
g(\varepsilon)=\frac{1}{1-\varepsilon_{m}} \quad \varepsilon_{m} \leq \varepsilon \leq 1.0
$$

Meanwhile the probability density for the maximum acceptable price (MAP) among the target group for pack $2, \quad p_{2}$, is given by

$$
h\left(p_{2}\right)=g\left(\varepsilon\left(p_{2}\right)\right)\left|\frac{\mathrm{d} \varepsilon}{\mathrm{d} p_{2}}\right| \quad 1 \leq p_{2} \leq p_{m}
$$

where the relationship between pack 2 price, $p_{2}$, and risk-aversion, $\varepsilon$, is found by combining Equation (46) and (51) to give:

$$
\varepsilon\left(p_{2}\right)=\frac{\ln \left(p_{2}+1\right)-\ln p_{2}}{\ln \left(p_{2}+1\right)}
$$

Differentiating Equation (60) with respect to $p_{2}$ 
gives $\mathrm{d} \varepsilon / \mathrm{d} p_{2}$ :

$$
\frac{\mathrm{d} \varepsilon}{\mathrm{d} p_{2}}=\frac{p_{2} \ln p_{2}-\left(p_{2}+1\right) \ln \left(p_{2}+1\right)}{p_{2}\left(p_{2}+1\right)\left(\ln \left(p_{2}+1\right)\right)^{2}}
$$

The resultant probability distribution, $h\left(p_{2}\right)$, is shown in Figure 3, for the case when $\rho_{m}=6, \varepsilon=0.101$. It is clear that the probability density for MAP falls away quickly as MAP increases.

The probability density for risk-aversion, $\varepsilon$, has also been modelled using the generalised, Double Power density [12], which provides an analytically tractable model for a variety of smooth distributions with a wide range of modes. Let

$$
\alpha=\varepsilon-\varepsilon_{m} \quad \varepsilon_{m} \leq \varepsilon \leq 1.0
$$

so that the minimum value of $\alpha$ is zero, while the maximum value, $\alpha_{m}$, of $\alpha$ is

$$
\alpha_{m}=1-\varepsilon_{m}
$$

The Double Power probability density for $\alpha$ is then defined on $\left(0, \alpha_{m}\right)$ by:

$$
f(\alpha)=a \alpha^{c}-b \alpha^{d} \quad 0 \leq \alpha \leq \alpha_{m}
$$

where, from [12]

$$
\begin{gathered}
a=\frac{(c+1)(d+1)}{(d-c) \alpha_{m}^{c+1}} \\
b=\frac{(c+1)(d+1)}{(d-c) \alpha_{m}^{d+1}}
\end{gathered}
$$

where $c$ and $d$ are free parameters to be specified by the user.

The corresponding probability density, $g(\varepsilon)$, for $\varepsilon$ is then

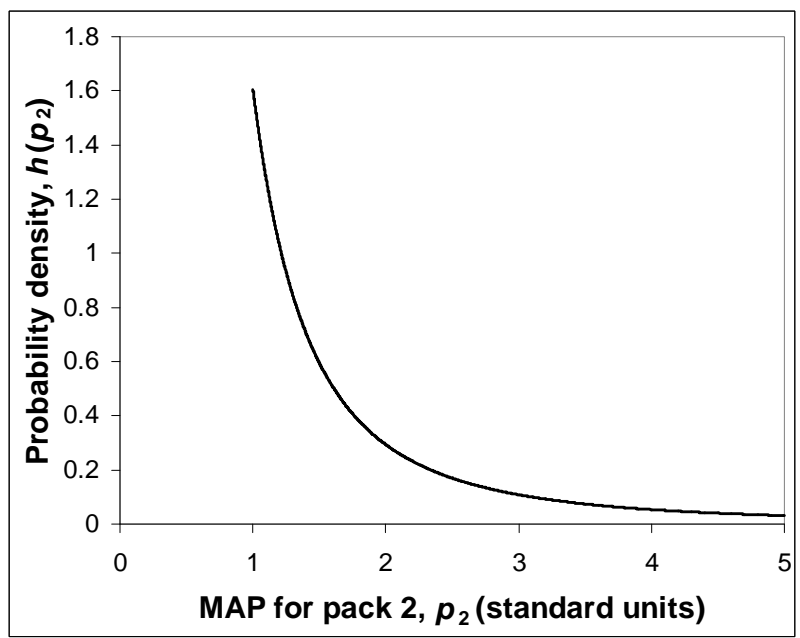

Figure 3. Probability distribution for maximum acceptable price for a uniform distribution of risk-aversion with $\varepsilon_{m}=\mathbf{0 . 1 0 1}$.

$$
g(\varepsilon)=f(\alpha(\varepsilon))\left|\frac{\mathrm{d} \alpha}{\mathrm{d} \varepsilon}\right|=a\left(\varepsilon-\varepsilon_{m}\right)^{c}-b\left(\varepsilon-\varepsilon_{m}\right)^{d}
$$

since $\mathrm{d} \alpha / \mathrm{d} \varepsilon=1$ from differentiating Equation (62).

Putting $c=1$ and $d=2$ produces a symmetrical distribution on $\varepsilon_{m} \leq \varepsilon \leq 1.0$, see Figure 4 , where the pack-size ratio has been set at $\rho_{m}=6$ so that $\varepsilon_{m}=0.101$. Applying Equations (59) and (61) gives the probability density for MAP for pack 2 given in Figure 5, showing how the probability density falls away for high values of MAP in a way similar to when the distribution is uniform over the same interval.

Skewed distributions for risk-aversion were produced by setting $c=1.0$ and varying the parameter, $d$, in the Double Power Equations (65) and (66). Setting $d=0.4$ produced a distribution skewed towards less caution,

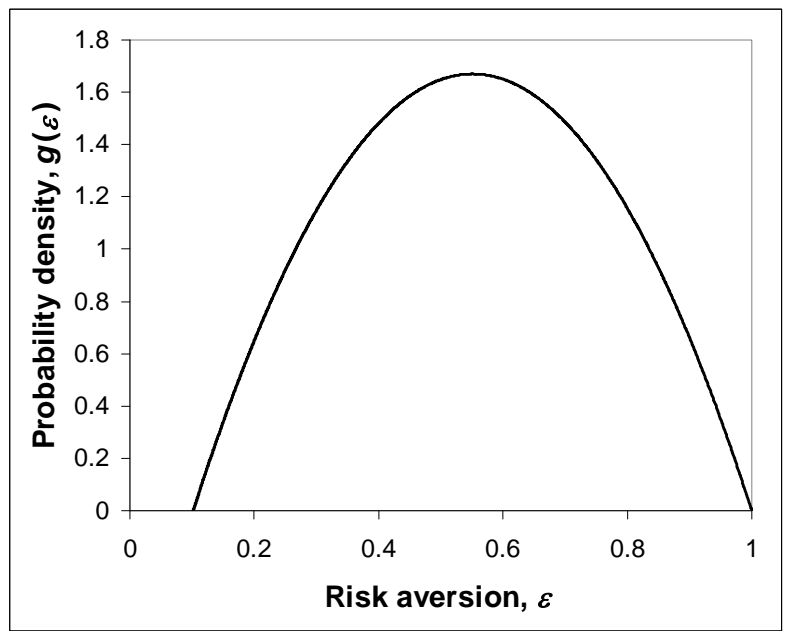

Figure 4. Double power probability distribution of riskaversion when $\varepsilon_{m}=0.101, c=1, d=2$.

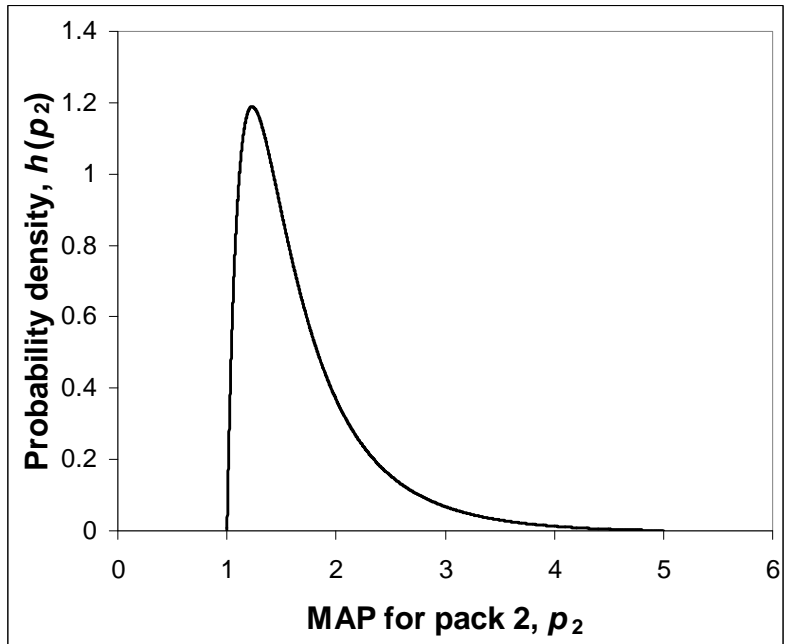

Figure 5. Probability distribution for maximum acceptable price for a symmetric double power distribution of riskaversion with $\varepsilon_{m}=0.101, c=1, d=2$. 
with a mode of $\varepsilon_{\text {mode }}=0.3$, as shown in Figure 6. A distribution skewed towards greater caution was found by putting $d=8$, with a resultant mode of $\varepsilon_{\text {mode }}=0.77$. See Figure 7. The probability density falls away for high values of MAP in both these cases also.

\subsection{The Optimal Largeness of Pack 2}

To facilitate the discussion of the results, we shall introduce the concept of the generosity of the offer, as measured by the price per unit of its contents. The price per unit for pack $2, \pi_{2}$, is given by:

$$
\pi_{2}=\frac{p_{2}}{L(2)}=\frac{L(2)-1}{L(2)}=1-\frac{1}{L(2)}
$$

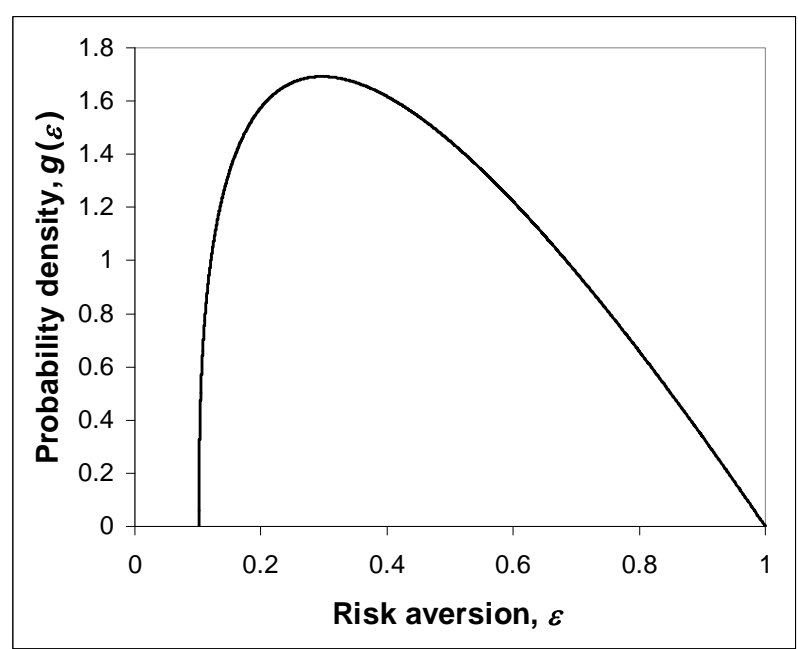

Figure 6. Double power probability distribution of riskaversion skewed towards less caution; $\varepsilon_{m}=0.101, c=1, d$ $=0.4$.

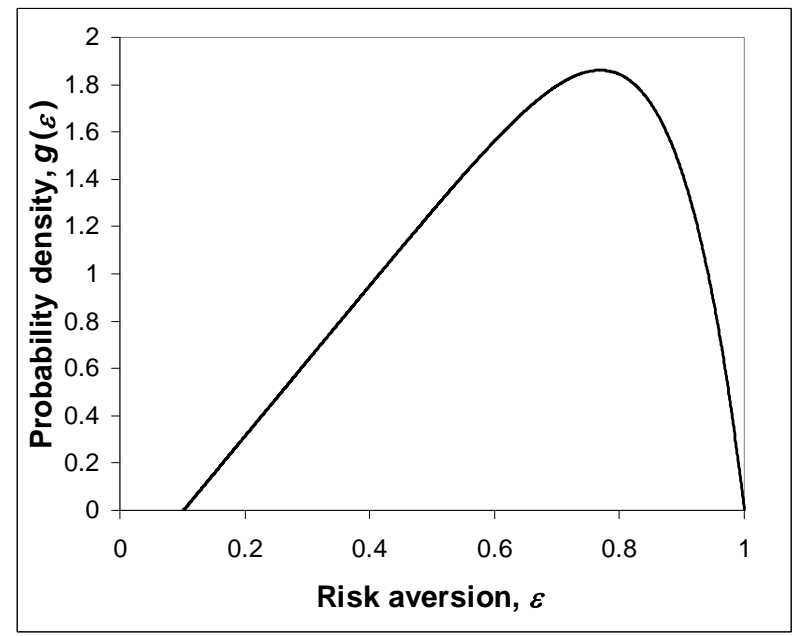

Figure 7. Double power probability distribution of riskaversion skewed towards greater caution; $\varepsilon_{m}=0.101, c=1$, $\boldsymbol{d}=\mathbf{8}$. where the price of pack 1 provides the base unit for price once again. Hence $\mathrm{d} \pi_{2} / \mathrm{d} L(2)=1 /(L(2))^{2}>0$, implying that increasing the largeness of pack 2 will raise the per-unit price, thus decreasing the generosity of the offer. In these terms, BOGOF is the most generous offer of those under consideration and 3 -for-2 is the least.

We may also note that the vendor will wish to cover at least the variable costs in any long-term offer. The money brought in per sale of pack 2 will be $p_{2}=L(2)-1$, while the variable cost associated with pack 2 will be $c_{v} L(2)$. Hence covering the variable cost of pack 2 requires that $L(2)-1-c_{v} L(2) \geq 0$ or

$$
L(2) \geq \frac{1}{1-c_{v}}
$$

Turning now to the problem of maximising profits, a solution may be found for the optimising Equation (56) in terms of $c_{v}$. The equation may be solved iteratively by first assigning a value to the optimal pack price, $p_{2}$, and then finding the variable cost, $c_{v}$, associated with pack 1 that reduces the left-hand side of the equation to zero. (The reverse process of fixing $c_{v}$ and finding the corresponding value of $p_{2}$ is equally valid, but tends to be less well conditioned.) The optimal largeness may then be found from the golden ratio, Equation (48), noting that $L(2)=\rho$.

Figure 8 shows the optimal largeness, $L(2)$, of pack 2 versus the variable cost, $c_{v}$, associated with pack 1 for four distributions for risk-aversion. It plots additionally the largeness of pack 2 needed to cover the variable cost (Equation (69)), which provides a base line. Clearly when $c_{v}=0.5$, selling 2 for the price of 1 (BOGOF) will cost 1 standard unit and bring in 1 standard unit, so that the variable cost of pack 2 is only just covered. When $c_{v}=0.667$, selling 3 for the price of 2 (BOGOF) will cost 2 standard units and bring in 2 standard units, just covering the variable cost of pack 2 again. Similar calculations will apply for all offers in between.

Let us consider the profit-maximising offer based on the assumption that the population follows a symmetrical Double Power probability density for risk-aversion with a lower level of $\varepsilon_{m}=0.369$ by the assumption that $\left.L(2)\right|_{\max }=3$. From Figure 8, the largeness offered when $c_{v}=0.5$ is just less than 2.3, which is less generous than the largeness of 2.0 which would just cover the variable costs. A largeness of 2.3 can sustain a profit because there are sufficient consumers in the target population prepared to pay 1.3 standard units or more for an appropriately sized pack 2 . When $c_{v}=0.6$, the optimal largeness has gone up to 2.65 , which may be compared with the largeness of 2.5 needed to cover variable costs. A largeness of 2.65 can be sustained because there are still enough consumers "left in" prepared to pay 1.65 standard units for the appropriately sized pack 2 . The 
margin between the optimal largeness and the variablecost-covering largeness has now reduced, however. This margin disappears altogether when the variable cost rises to $c_{v}=0.667$, when the optimal largeness of 3 will bring in only enough money to cover the variable costs. Figure 8 demonstrates how the optimal largeness associated with the Uniform distribution converges to the same point as that associated with the Double Power distribution. This convergence will be independent of the precise form of the distribution of risk-aversion above the lower limit of $\varepsilon_{m}=0.369$ that is consequential on $\left.L(2)\right|_{\max }=3$. While the vendor might want to set his largeness to a less generous value above 3 , there will be no consumer prepared to pay the correspondingly higher price, which would be more than 2 base units.

The vendor's plight will be eased if there are more people with lower risk-aversions in the target population. Thus when $\left.L(2)\right|_{\max }=6$ and so $\varepsilon_{m}=0.101$, the vendor will be able to set his largeness at less generous, higher values throughout the range. Thus he will be able to assign pack 2 a largeness of 3 for a variable cost, $c_{v}=0.59$, for the symmetrical Double Power density and for a variable cost, $c_{v}=0.50$, for a Uniform distribution over the same range.

Based on Figure 8, we may state generally that, if the vendor expects to be able to sell a 3-for-2 offer, so that $\left.L(2)\right|_{\max } \geq 3$, and requires that at least his variable costs should be covered, then the variable cost, $c_{v}$, associated with pack 1 must be at or below $66.7 \%$ of its selling price. More generally, a multibuy offer that is at least as generous as 3-for-2 cannot be profitable unless:

$$
0 \leq c_{v} \leq 0.667
$$

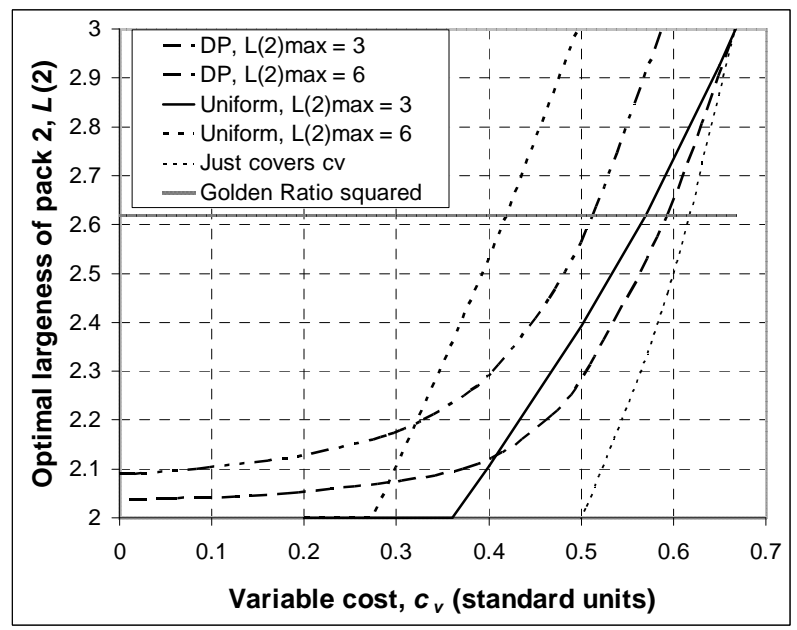

Figure 8. Optimal largeness of pack 2 vs. variable cost for Uniform and Double Power (DP) distributions for riskaversion, with $\left.L(2)\right|_{\max }=3$ and $\left.L(2)\right|_{\max }=6$. Also shown is the locus of the lowest largeness needed to cover the variable cost of pack 2 .
Figure 9 compares the effects of skewing the symmetrical Double Power distribution for risk-aversion towards lower caution and towards greater caution. The minimum risk-aversion is $\varepsilon_{m}=0.101$ in all three cases. The effect of a more cautious population of consumers is to lower the optimal largeness at any given $c_{v}$, while the optimal largeness of pack 2 increases when the population is less cautious.

Pinpointing the optimal largeness for pack 2 requires a knowledge of both $c_{v}$ and the distribution of risk-aversion in the target population. While $c_{v}$ may be determined accurately, the vendor is unlikely to know the exact form of the latter. It is argued in [19] that risk-aversion stays constant during any decision (whether to buy or not, in this instance), but nevertheless risk-aversion will vary with the importance of the decision. See also [22]. It is also likely to vary from person to person, dependent on both temperament and personal wealth. In the absence of more precise information, it is necessary to employ a range of credible distributions, leading to a plausible range of optimal largeness for a given variable cost, $C_{v}$.

The interval for $c_{v}$ for which it is sensible to consider multibuy offers in the range, $2 \leq L(2) \leq 3$ is given by condition (70). If the retailer is to restrict for simplicity his selection of the size and hence price of pack 2 to a ternary choice between BOGOF, Golden Ratio Pricing and 3-for-2, then, based on Figures 8 and 9, the following approximate $c_{v}$ ranges are appropriate for the three possible values of largeness for pack 2 :

$$
\begin{array}{ll}
0.0 \leq c_{v} \leq 0.4, & L(2)=2.0 \\
0.4<c_{v} \leq 0.6, & L(2)=\phi^{2} \approx 2.62 \\
0.6 \leq c_{v} \leq 0.667, & L(2)=3
\end{array}
$$

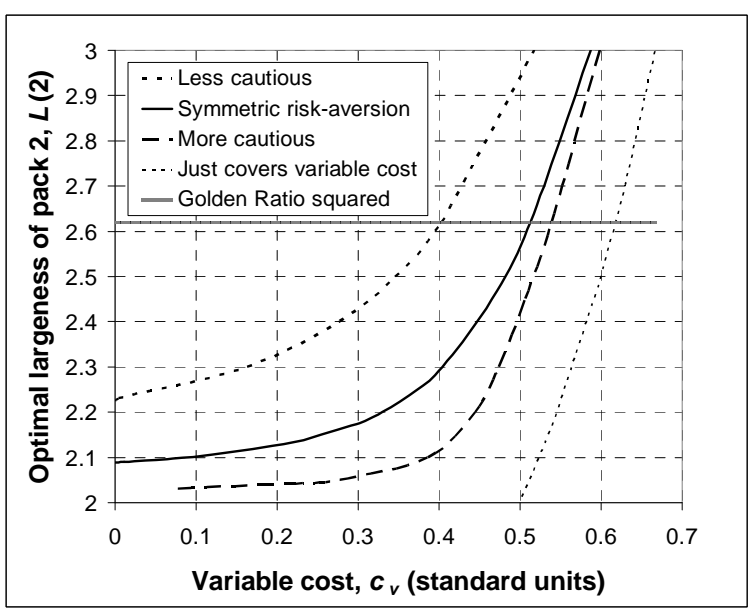

Figure 9. Optimal largeness of pack 2 vs. variable cost for Double Power distributions for risk-aversion, with $\left.L(2)\right|_{\max }=6$ with $c=1$ and $d=0.4$ (less cautious), $d=2$ (symmetric), and $d=8$ (more cautious). 
The curves suggest that the BOGOF provides the closest approach to optimality over roughly the lower three fifths of the range of variable cost where any of the three multibuy offers is able to generate a profit. The Golden Ratio will then be the best of the three offers in roughly the next three tenths of the profitable range of $c_{v}$, with 3 -for-2 becoming the optimal selection in roughly the highest decile. The boundaries suggested here are all approximate, as is clear from Figures $\mathbf{8}$ and $\mathbf{9}$, and should be regarded as indicative only.

Clearly if the entry pack contains a single, discrete item (a white shirt, for example), then fractional quantities, as implied by the Golden Ratio offer, will be impossible. Hence the vendor will need to adopt either a BOGOF or a 3-for-2 as an approximation.

The analysis suggests that the generous BOGOF can be justified on pure economic grounds only when the variable cost is below about $40 \%$ of the selling price of pack 1 . On the other hand, BOGOF will be attractive to the most cautious of the target market of cautious consumers, namely those with a risk-aversion, $\varepsilon=1$, and an offer that attracts the most cautious of the cautious consumers will also be attractive to rest. The BOGOF is thus ideal for eye-catching promotions that will appeal to all consumers who can be tempted by a multibuy offer. However, it should be offered on a temporary basis only unless the variable costs are low.

\section{Conclusions}

At a time when legal restrictions on quantity promotions are being given serious consideration in the UK, it is vital to have a proper understanding of multibuy promotions such as BOGOF. The theory of Relative Utility Pricing (RUP) developed in this paper takes account of the reaction of the customers to provide a quantitative economic explanation not only for supermarket promotional offers, but also for more general pricing of packs of different sizes in supermarkets and on the internet.

Grounded in standard economic thinking, the RUP model allows the derivation of a general formula that explains clearly and simply for the first time the two most widely used quantity promotions: BOGOF and 3 -for-the-price-of-2. It also provides a general explanation for why the price of a pack 2 that contains twice the amount of a commodity as the entry level pack, pack 1 , may need to be priced exactly the same to sell.

The paper has linked the RUP model to the theory of iso-elastic utility functions, allowing the relationship between risk-aversion and pack-size ratio to be stated. The same theoretical development also allows a simple relationship to be set down between risk-aversion and the demand elasticity for the product in question.

Successive doubling of pack sizes has been shown to correspond to a logarithmic utility function, where the risk-aversion is unity. This is associated with a neutral demand elasticity, viz. $\eta=-1$. Finite pack-size ratios greater than 2.0 are suitable for individuals with a risk-aversion value that lies above zero but no higher than unity - cautious consumers. They are also associated with a lower (more negative) values of demand elasticity: $\eta<-1$, which implies elastic demand.

Cautious consumers are shown to be the only sensible target for quantity promotions. There is no asymptotic limit to their gain in utility from additional quantities of a good, and they may be prepared to pay more for larger packs. Satisfying the desires of the average cautious consumer will result in a ratio of successive pack sizes equal to the square of the golden ratio, namely 2.62, while the price-ratio will be the golden ratio, 1.62. It is arguable that this pair of ratios is necessary in order to best satisfy the needs of consumers. Thus the golden ratio may be regarded as a marketing tool that vendors should consider using in the interests of their customers.

Golden Ratio Pricing can also be in the best interests of the vendor, as shown by an analysis of profit maximisation in the presence of the consumer attitudes reported in the paper. Promotions ranging from BOGOF through Golden Ratio Pricing to 3-for-the-price-of-2 can be profitable only if the variable cost of pack 1 is less than two thirds of the price of that pack. But higher profits are likely to come from Golden Ratio Pricing than from either BOGOF or 3-for-2 when variable costs as a fraction of pack 1 price lie in most of the upper half $(\sim 0.4$ to $\sim 0.6)$ of the range required to generate profit from any of these multibuy offers.

The RUP model has been applied to the prices of groceries, where it has been able to illuminate the pricing structure for "value" eggs in supermarkets. A significant degree of validation of the model comes from the closeness of its predictions both for pack size and price.

The application of the RUP model to the electronics sector has explained the non-obvious prices of USB memory sticks of increasing capacity. Linked to the binary system at the heart of every computer, the ingrained practice of the silicon chip industry to double the capacity of its products each year is enshrined in the influential Moore's Law [23]. This has the beneficial side-effect for the consumer that the price of the new product that is twice as fast or has twice the storage capacity as the old will settle down at the old price. Undoubtedly this has produced great benefits to the consumer. Although prima facie the practice may not be ideal for the vendor, it will be a driver for the exceptionally high rate of technological progress observed in the memory industry. Manufacturers are pressured to create higher capacity products due to the previous generation's prices quickly descending to commodity rates and thus returning low profits. 
Hence, the RUP model has explained the structure of quantity promotions, in terms of both the sizes of packs and the prices. Different packs should be able to command. This new theory should be of interest and value to vendors, consumers and regulators.

\section{Acknowledgements}

The authors would like to acknowledge numerous fruitful discussions during the preparation of the paper with Mr. Roger Jones, Honorary Fellow, City University London, and with Mr. Edward Ross and Dr Ian Waddington, both of Ross Technologies Ltd.

\section{REFERENCES}

[1] H. Wallop, "Buy-One-Get-One-Free Offers Are One of the Most Effective Marketing Tools in the Supermarket Industry," 2008.

http://www.telegraph.co.uk/news/uknews/2263645/Foodwaste-Why-supermarkets-will-never-say-bogof-to-buy-on e-get-one-free.html

[2] T. Evans, "The End of Buy One Get One Free? Government Considers Plans to Scrap Multibuy Deals to Tackle Obesity," This is Money.co.uk, 2013.

http://www.thisismoney.co.uk/money/news/article-23545 83/The-end-buy-one-free-Government-considers-plans-sc rap-multibuy-deals-tackle-obesity.html

[3] J. Koten, "Why Do Hot Dogs Come in Packs of 10 and Buns in 8s or 12s?" Wall Street Journal (Western Edition), 1984, p. 1.

[4] E. Gerstner and J. D. Hess, "Why Do Hot Dogs Come in Packs of 10 and Buns in 8 s or 12s? A Demand-Side Investigation," The Journal of Business, Vol. 60, No. 4, 1987, pp. 491-517. http://dx.doi.org/10.1086/296410

[5] A. Tabarrok, "Buy One Get One Free," 2004. http://www.marginalrevolution.com/marginalrevolution/2 004/03/buy_one_get_one.html

[6] M. W. Pressler, "Something Racy in the Male. Spilling Victoria's Secret, and Other Reader Correspondence," Washington Post, 2004, p. F05.

[7] T. Harford, "The Undercover Economist," Abacus, London, 2011.

[8] P. W. Dobson and E. Gerstner, "For a Few Cents More: Why Supersize Unhealthy Food," Marketing Science, Vol. 29, No. 4, 2010, pp. 770-778. http://dx.doi.org/10.1287/mksc.1100.0558

[9] D. Kahneman and A. Tversky, "Prospect Theory: An Analysis of Decision Under Risk," Econometrica, Vol. 47, No. 2, 1979, pp. 263-291.

\section{http://dx.doi.org/10.2307/1914185}

[10] R. H. Thaler, "Mental Accounting and Consumer Choice," Marketing Science, Vol. 4, No. 3, 1985, pp. 199-214. Republished 2008 in Marketing Science, Vol. 27, No. 1, 1985, pp. 15-25.

[11] P. Thomas and A. Chrystal, "Generalized Demand Densities for Retail Price Investigation," American Journal of Industrial and Business Management, Vol. 3, No. 3, 2013, pp. 279-294. http://dx.doi.org/10.4236/ajibm.2013.33034

[12] P. Thomas and A. Chrystal, "Retail Price Optimization from Sparse Demand Data," American Journal of Industrial and Business Management, Vol. 3, No. 3, 2013, pp. 295-306. http://dx.doi.org/10.4236/ajibm.2013.33035

[13] P. Thomas and A. Chrystal, "Using Relative Utility Pricing to Explain Prices in Supermarkets and on the Internet," American Journal of Industrial and Business Management, in Press.

[14] R. G. Lipsey and K. A. Chrystal, "An Introduction to Positive Economics," 8th Edition, Oxford University Press, Oxford, 1995.

[15] Amazon.co.uk, 2009. http://www.Amazon.co.uk/tag/usb\%20memory\%20stick/ products/ref=tag_tdp_sv_istp\#page $=2$ : sort $=$ relevant

[16] Mysupermarket.co.uk, 2009. http://www.mysupermarket.co.uk/Shopping/FindProducts .aspx?Query=Eggs

[17] J. W. Pratt, "Risk Aversion in the Small and in the Large," Econometrica, Vol. 32, No. 1-2, 1964, pp. 122136.

[18] K. J. Arrow, "Social Choice and Individual Values," John Wiley and Sons, New York, 1951.

[19] P. J. Thomas, "An Absolute Scale for Measuring the Utility of Money," Journal of Physics: Conference Series, Vol. 250, No. 1, 2010, Article ID: 012045. http://dx.doi.org/10.1088/1742-6596/250/1/012045

[20] H. M. Treasury, "Green Book," 2009. http://greenbook.treasury.gov.uk/annex05.htm

[21] T. Crilly, "50 Mathematical Ideas You Really Need to Know," Quercus, London, 2008.

[22] P. J. Thomas, R. D. Jones and W. J. O. Boyle, "The Limits to Risk Aversion. Part 2: The Permission Point and Examples," Process Safety and Environmental Protection, Vol. 88, No. 6, 2010, pp. 396-406. http://dx.doi.org/10.1016/j.psep.2010.07.001

[23] G. E. Moore, "Cramming More Components onto Integrated Circuits," Electronics, Vol. 38, No. 8, 1965, pp. 114-117. Reprinted Proceedings of the IEEE, Vol. 86, No. 1, 1998, pp. 82-85. http://en.wikipedia.org/wiki/Moore\%27s_law 


\section{Appendix A. Singular Homogeneity}

Consider the following transformation between two functions:

$$
H_{2}(m x)=m H_{1}(x) \text { for all numbers, } m
$$

The function, $H_{1}(x)$, may be seen to be transformed into the function, $H_{2}(m x)$, by multiplying both its input, $x$, and its output, $H_{1}(x)$, by the constant, $m$. Now suppose that $H_{1}($.$) is a homogeneous function of degree$ one. In this case, by the property of first-degree homogeneity:

$$
H_{1}(m x)=m H_{1}(x) \text { for all numbers, } m
$$

Linear operators possess this property of homogeneity of degree one. Comparing Equations (A.1) and (A.2), it is clear that if $H_{1}($.$) is a homogeneous function of degree$ one, then the second operator will be the same as the first: $H_{2}()=.H_{1}($.$) .$

Now consider Equation (7), which may be written in terms of a continuous variable for extent, $x$, as:

$$
\Delta u_{n 0}(L(n) x)=L(n) \Delta u_{10}(x) \quad 0 \leq x \leq b_{1}
$$

By Equation (A.3), the function, $\Delta u_{10}($.$) , may be$ transformed into the function, $\Delta u_{n 0}($.$) , by multiplying$ both the input, $x$, and the output, $\Delta u_{10}(x)$, by the constant, $L(n)$. Clearly Equation (A.3) bears a similarity to Equation (A.1), but it differs in the fact that Equation (A.3) applies only for the single value of $m: m=L(n)$. Hence we may describe Equation (A.3) as having the property of "singular homogeneity".

Under singular homogeneity, $\Delta u_{n 0}($.$) and \Delta u_{10}($. will be different, nonlinear functions, except for the limiting case when $\Delta u_{10}($.$) is linear in its argument. Such a$ situation will occur only when risk-aversion is zero, when the risk-neutral utility function emerges. (This contention becomes evident after substituting $\varepsilon=0$ into equations (A.5) and (A.6) below. At this point, the function, $\Delta u_{10}($.$) , has become homogeneous of degree 1, because$ the utility function is linear in its argument.)

An alternative but equivalent formulation of Equation (A.3) arises after replacing the extent variable, $x$, by a new variable of extent, $b$, where $b=L(n) x$ so that $x=b / L(n)$. Then equation (A.3) becomes:

$$
\Delta u_{n 0}(b)=L(n) \Delta u_{10}\left(\frac{b}{L(n)}\right) \quad 0 \leq b \leq L(n) b_{1}
$$

As an example, let us assume a pack-size ratio, $\rho=3$, as used by Sainsbury for its value eggs. From Equation (46), the matching risk-aversion is $\varepsilon=0.369$. Using the utility function given in Equation (37) to represent the utility that the person will gain from using the contents of pack 1 containing an amount, $b_{1}$, of good, $B$, we achieve, after noting that $L(1)=1$ :

$$
\Delta u_{10}(b)=\frac{b^{1-\varepsilon}-1}{1-\varepsilon}-\frac{-1}{1-\varepsilon}=\frac{b^{1-\varepsilon}}{1-\varepsilon}=1.585 b^{0.631} \quad 0 \leq b \leq b_{1}
$$

Meanwhile the utility of that the person or consortium of people will gain from using the contents of pack $n$ may be found by combining Equations (A.4) and (A.5) to give

$$
\begin{aligned}
\Delta u_{n 0}(b) & =L(n) \frac{1}{1-\varepsilon}\left(\frac{b}{L(n)}\right)^{1-\varepsilon}=(L(n))^{\varepsilon} \frac{b^{1-\varepsilon}}{1-\varepsilon} \\
& =(L(n))^{0.369} 1.585 b^{0.631} \quad 0 \leq b \leq L(n) b_{1}
\end{aligned}
$$

Figure 10 shows the utility gain from pack $1, \Delta u_{10}$, and that from pack $2, \Delta u_{20}$, plotted against the amount of the good, $B$, measured in standard units of the contents of back 1 , so that $b_{1}=1$ and $b_{2}=3$. These graphs might represent the case of one person buying pack 1 containing 6 eggs and a family unit of three buying pack 2 containing 18 eggs. The final utility gain for the individual, at $b=1$, will be 1.585 , while the final utility gain for the family of three will be $3 \times 1.585=4.755$, at $b=3$ standard units. The right-hand arrows on the graph show how this result may be derived by multiplying by the factor, $L(2)=3$, both the $x$ and the $y$ co-ordinates associated with the complete consumption of pack 1 .

Because Equation (A.3) has been formulated in continuous terms, we may apply the same transformation within the contents of pack 1 . Hence we may take the case where the individual has used $1 / 6$ of his pack 1 , one egg in this case, and multiply each of the co-ordinates of $\Delta u_{10}$, namely $(0.167,0.51)$ by a factor of three to give the corresponding co-ordinates $(0.5,1.53)$ of $\Delta u_{20}$. These show that the utility gained by the family of three in consuming half a standard unit, that is to say 3 eggs, is three times greater than that achieved by the single person eating one egg. The smaller arrows on the left-hand side of the graph indicate the graphical process.

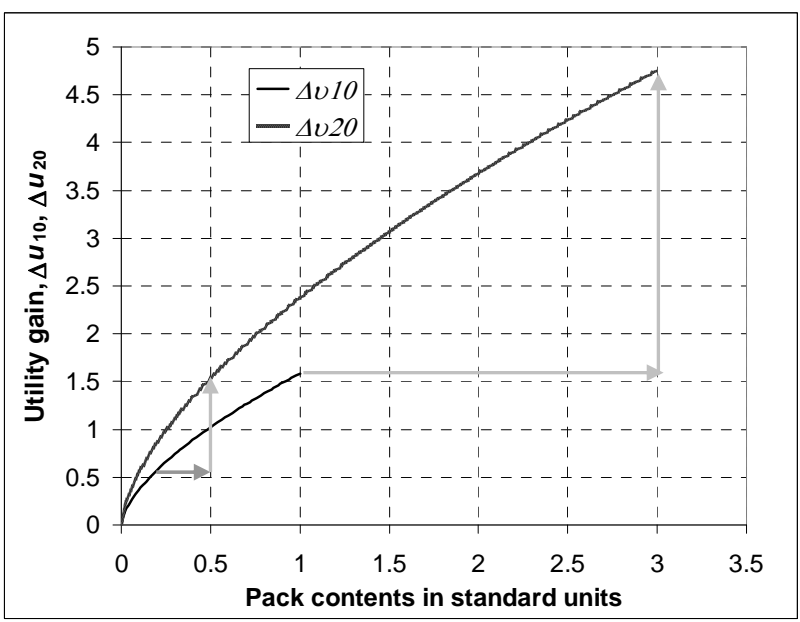

Figure 10. Graphical analysis of singular homogeneity. 


\section{Appendix B. The Range of Maximum Acceptable Price (MAP) for Pack $n$}

\section{B.1 The Highest MAP for Pack $n$}

Comparing Equations (3) and (17), it is clear that the price, $p_{n 1}$, resulting from a comparison of pack $n$ and pack 1 will be no greater than the price arising by comparing pack $n$ with pack 0 :

$$
p_{n 1} \leq p_{n 0} \quad \text { for } n>1
$$

It should be emphasised here that the comparison price, $p_{n k}$, arising from comparing pack $n$ with smaller packs will depend on the value of pack largeness and the achieved prices of for the lower packs, but not on the opinion of the individual, allowance for which comes from the weightings, $w_{k}^{(i)}$.

Putting $n=2$ into Equation (22) gives the individual's MAP for pack 2 as

$$
p_{2}^{(i)}=w_{0}^{(i)} p_{20}+w_{1}^{(i)} p_{21}
$$

By condition (B.1), $p_{21} \leq p_{20}$, and so, from equation (B.2), the highest value of the MAP, $p_{2}^{(i)}=p_{20}$, for any individual will occur when that person sets $w_{0}^{(i)}=1$ and $w_{1}^{(i)}=0$. Hence we may write:

$$
p_{2}^{(i)} \leq p_{20} \text { for all } i
$$

The achieved price, $p_{2}$, must be less than or equal to the highest MAP in the customer cohort, and so it follows that

$$
p_{2} \leq p_{20}
$$

where, from Equation (3),

$$
p_{20}=L(2) p_{1}
$$

We may now consider the highest MAP for pack 3 . Putting $k=2$ into Equation (20) and then using condition (B.4) gives the price of pack $n$ coming from a comparison with pack 2 as:

$$
\begin{aligned}
p_{n 2} & =\max \left(1.0, \frac{L(n)}{L(2)}-1\right) p_{2} \\
& \leq \max \left(1.0, \frac{L(n)}{L(2)}-1\right) L(2) p_{1} \\
& \leq \max \left(L(2) p_{1},(L(n)-L(2)) p_{1}\right) \\
& \leq \max \left(p_{20}, p_{n 0}-p_{20}\right) \quad n>2
\end{aligned}
$$

where Equation (B.5) has been used twice in the development. Clearly, for any strictly positive values,

$$
\begin{aligned}
& p_{20}, p_{n 0}: \\
& \max \left(p_{20}, p_{n 0}-p_{20}\right)<\max \left(p_{20}, p_{n 0}\right)
\end{aligned}
$$

But, by Equation (3), $p_{n 0}>p_{20}$, so that we may simplify the right-hand side of condition (B.7) to:

$$
\max \left(p_{20}, p_{n 0}-p_{20}\right)<p_{n 0}
$$

Substituting from condition (B.8) into condition (B.6), the price arising from the comparison of pack $n$ with pack 2 will obey:

$$
p_{n 2}<p_{n 0} \text { for } n>2
$$

If we put $n=3$ into Equation (22), the MAP for pack 3 emerges as

$$
p_{3}^{(i)}=w_{0}^{(i)} p_{30}+w_{1}^{(i)} p_{31}+w_{2}^{(i)} p_{32}
$$

Since by condition (B.1), $p_{31} \leq p_{30}$, and by condition (B.7), $p_{32}<p_{30}$, the highest value of any individual's MAP, $p_{3}^{(i)}$, will occur when $w_{0}=1, w_{1}=0$ and $w_{2}=0$, so that

$$
p_{3}^{(i)} \leq p_{30} \text { for all } i
$$

Moreover, since $p_{3} \leq p_{3}^{(i)}$, it follows that the achieved price for pack $3, p_{3}$, will obey

$$
p_{3} \leq p_{30}
$$

where, from equation (3),

$$
p_{30}=L(3) p_{1}
$$

It is clear that this process may be continued indefinitely, so that for any pack, $n \geq 1$, the upper limit of MAP will be defined by

$$
p_{n}^{(i)} \leq p_{n 0} \text { for all } i
$$

In other words the upper limit, $p_{n \max }$, for MAP for pack $n$ is given by:

$$
p_{n \max }=p_{n 0}=L(n) p_{1}
$$

\section{B.2 The Lowest MAP for Pack $n$}

A price for pack $n, p_{n}$, equal to the price of the next lowest pack, $p_{n-1}$, is possible, as demonstrated by the buy-one-get-one-free promotion and as explained above. However, bearing in mind the economic principle that more will be preferred to less, we may presume that the vendor will not allow the price, $p_{n}$, of pack $n$ to fall below the price, $p_{n-1}$, of pack $n-1$.

The lower price that the vendor would countenance, $p_{n}=p_{n-1}$, will have the effect that any potential customer for pack $n$ who has a MAP lower than $p_{n-1}$ will exclude himself from the cohort of customers of interest to the retailer. Thus the lowest MAP for pack $n$ will be simply the price, $p_{n-1}$, of the pack next down in size:

$$
p_{n \min }=p_{n-1}
$$

